Supplement of Geosci. Model Dev., 9, 223-245, 2016

http://www.geosci-model-dev.net/9/223/2016/

doi:10.5194/gmd-9-223-2016-supplement

(C) Author(s) 2016. CC Attribution 3.0 License.

(c) (i)

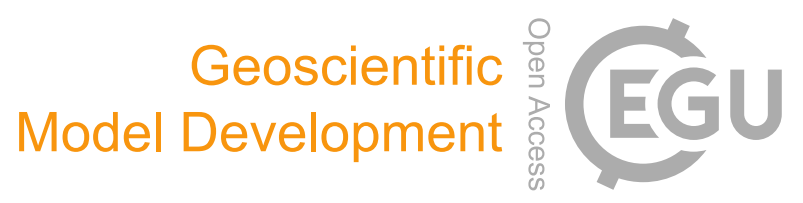

Supplement of

\title{
A multi-layer land surface energy budget model for implicit coupling with global atmospheric simulations
}

\section{J. Ryder et al.}

Correspondence to: J. Ryder (jryder@1sce.ipsl.fr)

The copyright of individual parts of the supplement might differ from the CC-BY 3.0 licence. 


\section{Supplementary material to: 'A multi-layer land surface energy budget for implicit coupling with global atmospheric simulations'}

\section{Contents}

S1 Notes 2

S2 Key equations and schemes 3

S2.1 Leaf vapour pressure assumption . . . . . . . . . . . . . . . . 3

S2.2 Physical and biophysical parameters: . . . . . . . . . . . . . 3

S2.3 The leaf energy balance equation for each layer . . . . . . . . . . . . . 4

S2.4 Sensible heat transport between each atmospheric layer . . . . . . . . . 4

S2.5 Latent heat transport between each atmospheric layer . . . . . . . . . 5

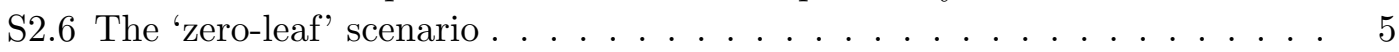

S2.7 Radiation scheme . . . . . . . . . . . . . . . . . . 6

S2.7.1 Longwave radiation . . . . . . . . . . . . . . . 6

S2.7.2 Shortwave radiation . . . . . . . . . . . . . 8

$\begin{array}{lr}\text { S3 Derivation of implicit solution } & \mathbf{1 0}\end{array}$

S3.1 Write equations in implicit format . . . . . . . . . . . . . . 10

S3.2 Implicit form of the energy balance equation . . . . . . . . . . . . . 10

S3.3 Implicit form of the sensible heat flux transport equation . . . . . . . . 10

S3.4 Implicit form of the latent heat flux transport equation . . . . . . . . . . 11

S3.5 Solving the leaf energy balance equation by induction . . . . . . . . . . 11

S3.6 Solving latent and sensible heat flux equations between layers by induction 13

S3.7 Alternative derivation . . . . . . . . . . . . . . . . . . . 19

$\begin{array}{lr}\text { S4 The boundary conditions } & 21\end{array}$

S4.1 The upper boundary conditions . . . . . . . . . . . . . . . . 21

S4.2 The lower boundary condition . . . . . . . . . . . . . . . . . 22 


\section{S1 Notes}

- Objective: This document expands on the model description present in the main text. We aimed at presenting a single documentation of the model. Therefore, we have duplicated parts of the methods here, to avoid multiple references to the main document.

- Symbolic notation: The symbolic notation used here is listed in full in Table 1 of the main text.

- Numbering of equations: the three 'key' equations (and variations as a result of substitution) are labelled a), b) and c) on the left hand side throughout the document. The assumptions are labelled in Roman numerals on the left hand side. All equations are numbered conventionally on the right hand side for ease of reference.

- Potential enthalpy: the present ORCHIDEE uses the term 'surface static energy' as the potential for calculating sensible heat flux. This is defined in the model (for the surface layer) as:

$$
p s_{\text {surf }}=C_{p}^{a i r} T_{\text {surf }}
$$

where $p s_{\text {surf }}$ is the surface static energy, $C_{p}^{a i r}$ is the mass specific heat capacity of air and $T_{\text {surf }}$ the surface temperature.

Now the enthalpy of a system $(\mathrm{H})$ is defined $H=U+p V$, but over the height of a surface model ( $<30 \mathrm{~m}$ approx), change in $p$ and $V$ is negligible, so:

$$
\begin{aligned}
\delta H & =\delta U+p \delta V+V \delta p \\
& =\left(\delta Q+\delta W+\delta W^{\prime}\right)+p \delta V+V \delta p
\end{aligned}
$$

(where we apply the first law of thermodynamics: $d U=\delta Q-\delta W$ )

now $\delta W=-p \delta V$, so we can say:

$$
\begin{aligned}
\delta H & =\delta Q+\delta W^{\prime}+V \delta p \\
& =Q+W^{\prime}+\int_{p_{0}}^{p} V \delta p \approx Q \\
& \approx C_{p}^{a i r} T
\end{aligned}
$$

So here we can also assume a proportional relationship between enthalpy and temperature over the vertical range of the model.

- Sign convention: For latent and sensible heat fluxes, an upward flux is positive (so a positive flux from the ground is cooling the ground) 


\section{S2 Key equations and schemes}

The structure of the derivation here outlined is based on that of the LMDz transport scheme (Dufresne \& Ghattas, 2009), but extended to include interactions with the vegetation layer at each level.

\section{S2.1 Leaf vapour pressure assumption}

The air within leaf level cavities is assumed completely saturated. This means that the vapour pressure of the leaf can be calculated as the saturated vapour pressure at that leaf temperature. Therefore the change in pressure within the leaf is assumed proportional to the difference in temperature between the present timestep and next timestep, multiplied by the rate of change in saturated pressure against temperature.

$$
\begin{aligned}
q_{0} \equiv q_{L, i}^{t+1} & =q_{s a t}^{T_{L, i}^{t}}+\left.\frac{\partial q_{s a t}}{\partial T}\right|_{T_{L, i}^{t}}\left(T_{L, i}^{t+1}-T_{L, i}^{t}\right) \\
& =\left.\frac{\partial q_{s a t}}{\partial T}\right|_{T_{L, i}^{t}}\left(T_{L, i}^{t+1}\right)+\left(q_{s a t}^{T_{L, i}^{t}}-\left.T_{L, i}^{t} \frac{\partial q_{s a t}}{\partial T}\right|_{T_{L, i}^{t}}\right) \\
& =\alpha_{i} T_{L, i}^{t+1}+\beta_{i}
\end{aligned}
$$

where $\alpha_{i}$ and $\beta_{i}$ are regarded as constants for each particular level and timestep so $\alpha_{i}=\left.\frac{\delta q_{\text {sat }}}{\delta T}\right|_{L, i} ^{t}$ and $\beta_{i}=\left(q_{s a t}^{T_{L, i}^{t}}-\left.T_{L, i}^{t} \frac{\partial q_{s a t}}{\partial T}\right|_{T_{L, i}^{t}}\right)$

But to find a solution we still need to find an expression for the terms $q_{s a t}^{T_{L, i}^{t}}$ and $\left.\frac{\partial q_{s a t}}{\partial T}\right|_{T_{L, i}^{t}}$ in $\alpha_{i}$ and $\beta_{i}$ above.

Using the empirical approximation of Tetens (e.g. as in Monteith and Unsworth (2008)) and the specific humidity vapour pressure relationship we can describe the saturation vapour pressure to within $1 \mathrm{~Pa}$ up to a temperature of about $35^{\circ} \mathrm{C}$.

\section{S2.2 Physical and biophysical parameters:}

We here concentrate on the formulation of an implicit solution that assumes a parameterisation for $R_{i}$ (the resistance to sensible heat flux at each level), $R_{i}^{\prime}$ (resistance to latent heat flux at each level) and $k_{i}$ (transport coefficients at each level). The derivation of these coefficients, based on literature study, will be described in a separate document. 


\section{S2.3 The leaf energy balance equation for each layer}

Now at the leaf level, we assume the energy balance for each layer. It is assumed that (for a leaf layer of volume $\Delta V_{i}$, area $\Delta A_{i}$ and thickness $\Delta h_{i}$ ):

$$
\Delta V_{i} \theta_{i} \rho_{v} \frac{d T_{\text {leaf,i }}}{d t}=\left(-H_{i}-\lambda E_{i}+R_{S W, i}+R_{L W, i}\right) \Delta A_{i}
$$

Dividing (S2.4) by $\Delta V_{i}$ :

$$
\theta_{i} \rho_{v} \frac{d T_{\text {leaf }, i}}{d t}=\left(-H_{i}-\lambda E_{i}+R_{S W, i}+R_{L W, i}\right)\left(\frac{1}{\Delta h_{i}}\right)
$$

The source sensible heat flux from the leaf at level $i$ is the difference between the leaf temperature and that above it, divided by $R_{i}$ which is the leaf resistance to sensible heat flux (a combination of stomatal and boundary layer resistance)). Similarly, the source latent heat flux from the leaf at level $i$ is the difference between the leaf temperature and that above it, divided by $R_{i}^{\prime}$ which is the leaf resistance to sensible heat flux. So the terms of (S2.5) are defined (in units $W / m^{2}$ ):

$$
\begin{gathered}
H_{i}=C_{p}^{a i r} \rho_{a} \frac{\left(T_{\text {leaf }, i}-T_{a, i}\right)}{R_{i}} \\
\lambda E_{i}=\lambda \rho_{a} \frac{\left(q_{\text {leaf }, i}-q_{a, i}\right)}{R_{i}^{\prime}}
\end{gathered}
$$

- $R_{L W}$ is the sum total of longwave radiation - that is: downwelling LW radiation from above the canopy, the LW radiation emitted from vegetation layer $i$ and the LW radiation reflected from the vegetation layers $i+1$ and $i-1$.

- $R_{S W}$ is the sum of short radiation, that is to say radiation downwelling on a level, but also that which is forward- of back- reflected from one level to another, or from the soil surface. So we express the sensible and latent heat fluxes between the leaf and the atmosphere respectively as:

a) $\theta_{i} \rho_{v} \frac{d T_{\text {leaf }, i}}{d t}=\left(-C_{p}^{a i r} \rho_{a} \frac{\left(T_{\text {leaf }, i}-T_{a, i}\right)}{R_{i}}-\lambda \rho_{a} \frac{\left(q_{\text {leaf }, i}-q_{a, i}\right)}{R_{i}^{\prime}}+R_{S W, i}+R_{L W(t o t), i}\right)\left(\frac{1}{\Delta h_{i}}\right)$

\section{S2.4 Sensible heat transport between each atmospheric layer}

The sensible heat flux profile is not constant over the height of the canopy. The rate of change of $T_{a, i}$ (the temperature of the atmosphere surrounding the leaf at level i) 
is proportional to the rate of change of sensible heat flux with height and the source sensible heat flux from the leaf at that level as in (S2.6) above:

b) $\quad C_{p}^{a i r} \rho_{a} \frac{d T_{a, i}}{d t} \Delta V_{i}=-\frac{\partial H_{a, i}}{\partial z} \Delta V_{i}+\left(\frac{T_{\text {leaf }, i}-T_{a, i}}{R_{i}}\right)\left(\frac{C_{p}^{a i r} \rho_{a}}{\Delta h_{i}}\right) \Delta V_{i}$

now $H_{a, i}=-\left(\rho_{a} C_{p}^{a i r}\right) k_{i} \frac{\partial T_{a, i}}{\partial z}$ (if the flux-gradient relation is assumed) so we can say:

b)

$$
\frac{d T_{a, i}}{d t} \Delta V_{i}=\frac{\partial}{\partial z}\left(k_{i} \frac{\partial \chi}{\partial z}\right) \Delta V_{i}+\left(\frac{T_{l e a f, i}-T_{a, i}}{R_{i}}\right)\left(\frac{1}{\Delta h_{i}}\right) \Delta V_{i}
$$

\section{S2.5 Latent heat transport between each atmospheric layer}

The latent heat flux profile is also not constant over the height of the canopy. The rate of change of $q_{a, i}$ (the specific humidity of the atmosphere surrounding the leaf at level i) is proportional to the rate of change of latent heat flux with height and the source latent heat flux from the leaf as in (S2.7):

$$
\begin{aligned}
\lambda \rho_{a} \frac{d q_{a, i}}{d t} \Delta V_{i} & =-\frac{\partial(\lambda E)_{a, i}}{\partial z} \Delta V_{i}+\left(\frac{q_{\text {leaf }, i}-q_{a, i}}{R_{i}^{\prime}}\right)\left(\frac{\lambda \rho_{a}}{\Delta h_{i}}\right) \Delta V_{i} \\
& =-\frac{\partial(\lambda E)_{a, i}}{\partial z} \Delta V_{i}+\left(\frac{\left(\alpha T_{\text {leaf }, i}+\beta_{i}\right)-q_{a, i}}{R_{i}^{\prime}}\right)\left(\frac{\lambda \rho_{a}}{\Delta h_{i}}\right) \Delta \text { (S\& }
\end{aligned}
$$

now $(\lambda E)_{a, i}=-\left(\lambda \rho_{a}\right) k_{i} \frac{d q_{a, i}}{d z}$ (again assuming the flux-gradient relation) so:

$$
\text { c) } \frac{d q_{a, i}}{d t} \Delta V_{i}=\frac{\partial}{\partial z}\left(k_{i} \frac{\partial q_{a, i}}{\partial z}\right) \Delta V_{i}+\left(\frac{\left(\alpha T_{\text {leaf }, i}+\beta_{i}\right)-q_{a, i}}{R_{i}^{\prime}}\right)\left(\frac{1}{\Delta h_{i}} \Delta V_{i}\right)
$$

\section{S2.6 The 'zero-leaf' scenario}

Canopy layers that do not contain foliage may be accounted for at a level by assuming that $R_{i}=R_{i}^{\prime}=\infty$ for that level (i.e. an open circuit), and that the various coefficients 
that relate to the leaf interactions at that level $\left(R_{S W}, R_{L W}, C_{T, i}, C_{T, i+1}, C_{q, i}, C_{q, i+1}\right.$, $\left.D_{i}, E_{i}, F_{i}, D_{i+1}, E_{i+1}, F_{i+1}\right)$ are zero.

\section{S2.7 Radiation scheme}

The radiation approach is the application of the longwave Radiation Transfer Matrix (LRTM) (Gu (1988); Gu et al. (1999)), as applied in Ogée et al. (2003). This approach separates the calculation of the radiation distribution completely from the implicit expression. Instead a single source term for the longwave radiation is added at each level. This means that the distribution of radiation is no longer completely explicit (i.e. an explicit scheme makes use of information only from the 'present' and not the 'next' time step). However, an advantage of the approach is that it accounts for a higher order of reflections from adjacent levels than the single order that is assumed in the process above.

The components for longwave radiation are abbreviated as:

$$
R_{L W, i}=\eta_{1, i} T_{l e a f, i}^{t+1}+\eta_{2, i}
$$

The shortwave radiation component is abbreviated as:

$$
R_{S W, i}=\eta_{3, i} R_{S W}^{\text {down }}
$$

where $\eta_{1, i}, \eta_{2, i}$ and $\eta_{3, i}$ are components of the radiation scheme. $\eta_{1, i}$ accounts for the components relating to emission and absorption of LW radiation from the vegetation at level $i$ (i.e. the implicit parts of the longwave scheme relating to the level $i$ ) and $\eta_{2, i}$ the components relating to radiation from vegetation at all other levels incident on the vegetation at level $i$ (i.e. the non-implicit part of the longwave scheme), as well as explicit parts from the level $i$.

$\eta_{3, i}$ is the component of the SW radiation scheme - it describes the fraction of the total downwelling shortwave light that is absorbed at each layer, including over multiple forward- and back-reflections, as simulated by the multilayer albedo scheme (McGrath et al., 2015). The fraction of original downwelling SW radiation that is ultimately reflected from the surface and from the vegetation cover back to the canopy can then be calculated using this information.

\section{S2.7.1 Longwave radiation}

We applied a version of the Longwave Radiation Transfer Scheme of Gu (1988, 1999), with some modifications that are summarised here. The method assumes that scattering coefficients for longwave radiation are very small (of the order of 0.05), and can thus be 
ignored. The basics of the scheme can be described by the matrix equation for a canopy of m levels:

$$
\left(\begin{array}{c}
\Delta \aleph_{\text {surf }} \\
\Delta \aleph_{1} \\
\cdot \\
\cdot \\
\cdot \\
\Delta \aleph_{m} \\
\Delta \aleph_{\text {above }}
\end{array}\right)=\left(\begin{array}{ccccccc}
\alpha_{0,0}^{L W} & \alpha_{0,1}^{L W} & . & . & . & \alpha_{0, m}^{L W} & \alpha_{0, m+1}^{L W} \\
\alpha_{1,0}^{L W} & \alpha_{1,1}^{L W} & \cdot & . & \cdot & \alpha_{1, m}^{L W} & \alpha_{1, m+1}^{L W} \\
\cdot & & & & & \cdot \\
\cdot & & & & & \cdot \\
\cdot & & & & & \cdot \\
\alpha_{m, 0}^{L W} & \alpha_{m, 1}^{L W} & \cdot & \cdot & \cdot & \alpha_{m, m}^{L W} & \alpha_{m, m+1}^{L W} \\
\alpha_{m+1,0}^{L W} & \alpha_{m+1,1}^{L W} & \cdot & \cdot & \cdot & \alpha_{m+1, m}^{L W} & \alpha_{m+1, m+1}^{L W}
\end{array}\right)\left(\begin{array}{c}
\sigma\left(T_{\text {surf }}^{t}\right)^{4} \\
\sigma\left(T_{\text {leaf }, 1}^{t}\right)^{4} \\
\cdot \\
\cdot \\
\cdot \\
\sigma\left(T_{\text {leaf }, m}^{t}\right)^{4} \\
R_{L W}
\end{array}\right)
$$

for which each element $\alpha_{i, j}^{L W}$ is defined as:

$$
\alpha_{i, j}= \begin{cases}-1, & \mathrm{i}=\mathrm{j}=0, \\ \Im\left(\ell_{t}-\ell_{j-1}\right)-\Im\left(\ell_{t}-\ell_{j}\right), & \mathrm{i}=0, \mathrm{j}=1,2, \ldots, \mathrm{m} \\ \Im\left(\ell_{t}\right), & \mathrm{i}=0, \mathrm{j}=\mathrm{m}+1 \\ \Im\left(\ell_{j}-\ell_{i-1}\right)-\Im\left(\ell_{j-1}-\ell_{i-1}\right)-\Im\left(\ell_{j}-\ell_{i}\right)+\Im\left(\ell_{j-1}-\ell_{i}\right), & \mathrm{i}=1,2, \ldots, \mathrm{m}, \mathrm{j}=1,2, \ldots, \mathrm{i}-1 \\ 2 \Delta \Im\left(\ell_{i}\right)-2, & \mathrm{i}=1,2, \ldots, \mathrm{m}, \mathrm{j}=\mathrm{i} \\ \Im\left(\ell_{i}-\ell_{j-1}\right)-\Im\left(\ell_{i}-\ell_{j}\right)-\Im\left(\ell_{i-1}-\ell_{j-1}\right)+\Im\left(\ell_{i-1}-\ell_{j}\right), & \mathrm{i}=1,2, \ldots, \mathrm{m}, \mathrm{j}=\mathrm{i}+1, \mathrm{i}+2, \ldots ., \mathrm{m} \\ \Im\left(\ell_{t}\right), & \mathrm{i}=\mathrm{m}+1, \mathrm{j}=0 \\ \Im\left(\ell_{j}\right)-\Im\left(\ell_{j-1}\right), & \mathrm{i}=\mathrm{m}+1, \mathrm{j}=1,2, \ldots, \mathrm{m} \\ -1, & \mathrm{i}=\mathrm{m}+1, \mathrm{j}=\mathrm{m}+1 .\end{cases}
$$

Now, the column on the left hand side of the expression $\Delta \aleph_{i}$ represents the net longwave radiation that is absorbed at each level vegetation $i$, as well as the soil surface layer $\left(\aleph_{\text {surf }}\right)$ and the atmosphere directly above the canopy $\left(\aleph_{\text {above }}\right) . T_{i}$, is the temperature of each layer, and $R_{L W}$ represents the downwelling longwave radiation from above the canopy. Here $\ell_{i}$ represents the cumulative leaf area index when working up to level $i$ from the ground, that is to say calculated as:

$$
\ell_{i}=\sum_{1}^{i} L A I_{i}
$$

The function $\Im(\ell)$ simulates the effect of canopy structure on the passage of longwave radiation, and is defined as: 


$$
\Im(\ell)=2 \int_{0}^{1} e^{-\frac{\ell G_{l e a f}(\mu)}{\mu}} \mu d \mu
$$

$G_{\text {leaf }}(\mu)$ is a function that represents the orientation of the leaves. $\Im(\ell)$ is then solved from integrations. So multiplying out the terms, we have the an expression for $\Delta \aleph$ at each level:

$$
\begin{aligned}
& \Delta \aleph=\alpha_{i, 0}^{L W} \sigma\left(T_{\text {surf }}^{t}\right)^{4}+\alpha_{i, 1}^{L W} \sigma\left(T_{\text {leaf }, 1}^{t}\right)^{4} \ldots, \ldots+\alpha_{i, i}^{L W} \sigma\left(T_{\text {leaf }, i}^{t}\right)^{4} \\
& \ldots, \ldots+\alpha i, m^{L W} \sigma\left(T_{\text {leaf }, m}^{t}\right)^{4}+\alpha_{L W}^{i, m+1} R_{L W}
\end{aligned}
$$

This part of the energy budget model is explicit, relying on temperature at the last time step. However, for the level $i$ in each case we can make the expression semi-implicit, by expressing partly in terms of the leaf temperature at the next time step, through use a truncated Taylor expansion, such that:

$$
\begin{aligned}
\alpha_{i, i}^{L W} \sigma\left(T_{\text {leaf }, i}^{t+1}\right)^{4} & \approx \alpha_{i, i}^{L W} \sigma\left(\left(T_{\text {leaf }, i}^{t}\right)^{4}+4\left(T_{\text {leaf }, i}^{t}\right)^{3}\left(T_{\text {leaf }, i}^{t+1}-T_{\text {leaf }, i}^{t}\right)\right) \\
& =\alpha_{i, i}^{L W} \sigma\left(4\left(T_{\text {leaf }, i}^{t}\right)^{3} T_{\text {leaf }, i}^{t+1}-3\left(T_{\text {leaf }, i}^{t}\right)^{4}\right)
\end{aligned}
$$

so, in effect, (S2.19) can be expressed as:

$$
\begin{array}{r}
\Delta \aleph=\alpha_{i, 0}^{L W} \sigma\left(T_{\text {surf }}^{t}\right)^{4}+\alpha_{i, 1}^{L W} \sigma\left(T_{\text {leaf }, 1}^{t}\right)^{4} \ldots, \ldots+\alpha_{i, i}^{L W} \sigma\left(4\left(T_{\text {leaf }, i}^{t}\right)^{3} T_{\text {leaf }, i}^{t+1}-3\left(T_{\text {leaf }, i}^{t}\right)^{4}\right) \\
\ldots, \ldots+\alpha i, m^{L W} \sigma\left(T_{\text {leaf }, m}^{t}\right)^{4}+\alpha_{L W}^{i, m+1} R_{L W} \quad(\mathrm{~S} 2.22)
\end{array}
$$

and so we calculate the matrix (44) above with the central diagonal for which $i=j$ set to zero and designate the coefficients (S2.14) as:

$$
\begin{aligned}
& \eta_{1, i}=\alpha_{i, i}^{L W} \sigma\left(T_{\text {leaf }, i}^{t}\right)^{3} \\
& \eta_{2, i}=\aleph_{i}-3 \alpha_{i, i}^{L W} \sigma\left(T_{\text {leaf }, i}^{t}\right)^{4}
\end{aligned}
$$

\section{S2.7.2 Shortwave radiation}

We implement the scheme from McGrath et al. (2015), which is a development of Pinty et al. (2006). The scheme accounts for three-dimensional canopies through use of a domain-averaged structure factor (the effective Leaf Area Index). To summarise, in this 
approach the SW radiation is divided into several terms at each level expressed as a fraction of the total SW downwelling radiation, as listed below.

We use the notation $\psi$ to denote the fraction of the above canopy SW radiation that is absorbed $\left(\psi_{i}^{a b s}\right)$, is incoming to each level $i$ either by direct transmission (uncollided) or by reflection (collided). The symbol ' $\downarrow$ ' refers to the sum of all downwelling shortwave radiation (i.e. directly transmitted radiation, and second order reflected radiation), whilst ' $\uparrow$ ' refers to the sum of all upwelling shortwave radiation (i.e. sum of first-order and second-order reflected radiation from all levels).

- $\psi_{i, \downarrow \text {,out }}^{\text {unclided }}$ - fraction corresponding to uncollided light transmitted through level $i$ without striking any element. This is also described as 'unscattered, collimated radiation'.

- $\psi_{i, \downarrow}^{\text {collided }}$ - fraction corresponding to collided, transmitted light transmitted through level $i$ after striking vegetation one or more times. This is also described as 'forward scattered isotropic radiation'.

- $\psi_{i, \uparrow}^{\text {collided }}$ - fraction corresponding to collided light reflected upwards after striking vegetation one or more times. This is also described as 'back scattered isotropic radiation'

Now, using these probabilities of the fate of the light, the equations of Pinty et al. (2006) are applied to each layer of the canopy in turn, initially for the top layer, with the assumption of a black background underneath. Some of the flux is reflected back into the atmosphere, some absorbed, and some transmitted or forward scattered into the level below. The nature of the light (collimated or isotropic) determines how it interacts with the canopy, so these two types of light are accounted for separately in the model. The calculations are repeated for this lower level, with this fraction of the light. Calculations through all of the levels are continued as an iterative process until all light is accounted for through either reflection (or back scatter) back to the atmosphere or absorption by the vegetation or by the soil. McGrath et al. (2015) provides full details of the iteration scheme, which produces as output $\psi_{i}^{a b s}$, which is the fraction of the incident SW at the top of the canopy that is absorbed at each level at the end of the convergence loop. Tests in that work demonstrate that results from a multi-level case are in acceptable agreement with those from the single level.

Over the canopy vegetation levels, we can now define the coefficient $\eta_{3, i}$ in equation (S2.15):

$$
\begin{aligned}
& \eta_{3, i}=\psi_{i}^{a b s} \\
& \eta_{3, \text { surf }}=\psi_{\text {surface }}^{a b s}
\end{aligned}
$$




\section{S3 Derivation of implicit solution}

\section{S3.1 Write equations in implicit format}

To maintain the implicit coupling between the atmospheric model (i.e. LMDZ) and the land surface model (i.e. ORCHIDEE) we need to express the relationships that are outlined above in terms of a linear relationship between the 'present' timestep $t$ and the 'next' timestep $t+1$.

We therefore re-cast equations a), b) and c) in implicit form (i.e. in terms of the 'next' timestep, which is $t+1$, as below.

\section{S3.2 Implicit form of the energy balance equation}

We substitute the expressions (S2.14) and (S2.15) to the energy balance equation (S2.5), which we rewrite in implicit form:

$$
\begin{array}{r}
\theta_{i} \rho_{v} \frac{\left(T_{\text {leaf }, i}^{t+1}-T_{\text {leaf }, i}^{t}\right)}{\Delta t}=\left(\frac{1}{\Delta h_{i}}\right)\left(-C_{p}^{a i r} \rho_{a} \frac{\left(T_{\text {leaf }, i}^{t+1}-T_{a, i}^{t+1}\right)}{R_{i}}\right. \\
\left.-\lambda \rho_{a} \frac{\left(\alpha_{i} T_{\text {leaf }, i}^{t+1}+\beta_{i}-q_{a, i}^{t+1}\right)}{R_{i}^{\prime}}+\eta_{1} T_{\text {leaf }, i}^{t+1}+\eta_{2}+\eta_{3} R_{S W}^{d o w n}\right)
\end{array}
$$

Rearranging to isolate the state variables terms (temperature and specific humidity) at the 'next' timestep:

$$
\begin{array}{r}
T_{\text {leaf }, i}^{t+1}-T_{\text {leaf }, i}^{t}=\frac{\lambda \rho_{a} \Delta t \beta_{i}}{\left(\rho_{v} \Delta h_{i}\right) R_{i}^{\prime} \theta_{i}}+\frac{\eta_{4} R_{S W}^{\text {down }} \Delta t}{\left(\rho_{v} \Delta h_{i}\right) \theta_{i}}+\frac{\eta_{1} R_{L W}^{\text {down }} \Delta t}{\left(\rho_{v} \Delta h_{i}\right) \theta_{i}}+\frac{\eta_{3} \Delta t}{\left(\rho_{v} \Delta h_{i}\right) \theta_{i}} \\
+T_{\text {leaf }, i}^{t+1}\left(-C_{p}^{\text {air }} \rho_{a} \frac{\Delta t}{\left(\rho_{v} \Delta h_{i}\right) R_{i} \theta_{i}}-\lambda \rho_{a} \frac{\Delta t \alpha_{i}}{\left(\rho_{v} \Delta h_{i}\right) R_{i}^{\prime} \theta_{i}}+\frac{\eta_{2} \Delta t}{\left(\rho_{v} \Delta h_{i}\right) \theta_{i}}\right) \\
+T_{a, i}^{t+1} C_{p}^{\text {air }} \rho_{a}\left(\frac{\Delta t}{R_{i} \theta_{i}\left(\rho_{v} \Delta h_{i}\right)}\right)+q_{a, i}^{t+1} \lambda \rho_{a}\left(\frac{\Delta t}{\left(\rho_{v} \Delta h_{i}\right) R_{i}^{\prime} \theta_{i}}\right)
\end{array}
$$

\section{S3.3 Implicit form of the sensible heat flux transport equation}

We difference (S2.10), and divide by $\Delta V_{i}$ : 


$$
\text { b) } \begin{aligned}
\frac{T_{a, i}^{t+1}-T_{a, i}^{t}}{\Delta t}=k_{i}\left(\frac{\left(T_{a, i+1}^{t+1}-T_{a, i}^{t+1}\right)}{\Delta z_{i} \Delta h_{i}}\right)-k_{i-1} & \left(\frac{\left(T_{a, i}^{t+1}-T_{a, i-1}^{t+1}\right)}{\Delta z_{i-1} \Delta h_{i}}\right) \\
& +\left(\frac{1}{\Delta h_{i}}\right) \frac{\left(T_{l e a f, i}^{t+1}-T_{a, i}^{t+1}\right)}{R_{i}}
\end{aligned}
$$

\section{S3.4 Implicit form of the latent heat flux transport equation}

We difference (S2.13), and divide by $\Delta V_{i}$ :

$$
\text { c) } \begin{aligned}
\frac{q_{a, i}^{t+1}-q_{a, i}^{t}}{\Delta t}=k_{i}\left(\frac{\left(q_{a, i+1}^{t+1}-q_{a, i}^{t+1}\right)}{\Delta z_{i} \Delta h_{i}}\right) & -k_{i-1}\left(\frac{\left(q_{a, i}^{t+1}-q_{a, i-1}^{t+1}\right)}{\Delta z_{i-1} \Delta h_{i}}\right) \\
& +\left(\frac{1}{\Delta h_{i}}\right) \frac{\left(\alpha_{i} T_{\text {leaf }, i}^{t+1}+\beta_{i}-q_{a, i}^{t+1}\right)}{R_{i}^{\prime}}
\end{aligned}
$$

\section{S3.5 Solving the leaf energy balance equation by induction}

We determine to solve these equations by assuming a solution of a particular form and finding the coefficients that are introduced in terms of the coefficients of the layer above. This is 'proof by induction'. Now, for (S3.2) we want to express $T_{a, i}^{t+1}$ in terms of values further down the column, to allow the equation to solved by 'moving up' the column, as in Richtmyer and Morton (1967) and Dufresne and Ghattas (2009).

We assume that:

$$
\begin{aligned}
& \text { i) } T_{a, i}^{t+1}=A_{T, i} T_{a, i-1}^{t+1}+B_{T, i}+C_{T, i} T_{\text {leaf }, i}^{t+1}+D_{T, i} q_{a, i-1}^{t+1} \\
& \text { ii) } q_{a, i}^{t+1}=A_{q, i} q_{a, i-1}^{t+1}+B_{q, i}+C_{q, i} T_{\text {leaf }, i}^{t+1}+D_{q, i} T_{a, i-1}^{t+1}
\end{aligned}
$$

These two expressions are the equivalent of equation 11.7 (from Richtmyer and Morton (1967)) for the present system.

We also re-write these expressions in terms of the values of the next level: 
i) $T_{a, i+1}^{t+1}=A_{T, i+1} T_{a, i}^{t+1}+B_{T, i+1}+C_{T, i+1} T_{l e a f, i+1}^{t+1}+D_{T, i+1} q_{a, i}^{t+1}$

ii) $q_{a, i+1}^{t+1}=A_{q, i+1} q_{a, i}^{t+1}+B_{q, i+1}+C_{q, i+1} T_{l e a f, i+1}^{t+1}+D_{q, i+1} T_{a, i}^{t+1}$

where $A_{T, i}, B_{T, i}, C_{T, i}, D_{T, i}, A_{q, i}, B_{q, i}, C_{q, i}$ and $D_{q, i}$ are constants for that particular level and timestep but are (as yet) unknown. We thus substitute (S3.5) and (S3.6) into (S3.2) to eliminate $T_{a, i}^{t+1}$

$$
\begin{aligned}
T_{\text {leaf }, i}^{t+1}-T_{\text {leaf }, i}^{t} & =-\lambda \rho_{a} \frac{\Delta t \beta_{i}}{\left(\rho_{v} \Delta h_{i}\right) R_{i}^{\prime} \theta_{i}}+\frac{\eta_{2} \Delta t}{\left(\rho_{v} \Delta h_{i}\right) \theta_{i}} \\
& +\frac{\eta_{3} R_{S W}^{d o w n} \Delta t}{\left(\rho_{v} \Delta h_{i}\right) \theta_{i}} \\
& +\frac{T_{L, i}^{t+1}}{\left(\rho_{v} \Delta h_{i}\right)}\left(-\frac{\lambda \rho_{a} \Delta t \alpha_{i}}{R_{i}^{\prime} \theta_{i}}-\frac{C_{p}^{a i r} \rho_{a} \Delta t}{\theta_{i} R_{i}}+\frac{\eta_{1} \Delta t}{\theta_{i}}\right) \\
& +\left(\frac{C_{p}^{a i r} \rho_{a} \Delta t}{\left(\rho_{v} \Delta h_{i}\right) R_{i} \theta_{i}}\right)\left(A_{T, i} T_{a, i-1}^{t+1}+B_{T, i}+C_{T, i} T_{\text {leaf }, i}^{t+1}+D_{T, i} q_{a, i-1}^{t+1}\right) \\
& +\left(\frac{\lambda \rho_{a} \Delta t}{\left(\rho_{v} \Delta h_{i}\right) R_{i}^{\prime} \theta_{i}}\right)\left(A_{q, i} q_{a, i-1}^{t+1}+B_{q, i}+C_{q, i}\left(T_{\text {leaf }, i}^{t+1}\right)+D_{q, i} T_{a, i-1}^{t+1}\right)
\end{aligned}
$$

or, to rearrange again in terms of the unknown state variables (left hand side) and the know variables (right hand side):

$$
\begin{gathered}
T_{L, i}^{t+1}\left(1-\frac{\Delta t C_{p}^{a i r}}{\left(\rho_{v} \Delta h_{i}\right) R_{i} \theta_{i}} C_{T, i}+\frac{\lambda \rho_{a} \alpha_{i} \Delta t}{\left(\rho_{v} \Delta h_{i}\right) R_{i}^{\prime} \theta_{i}}-\frac{\eta_{1} \Delta t}{\left(\rho_{v} \Delta h_{i}\right) \theta_{i}}\right. \\
\left.+\frac{C_{p}^{a i r} \rho_{a} \Delta t}{\left(\rho_{v} \Delta h_{i}\right) \theta_{i} R_{i}}-\frac{\lambda \rho_{a} \Delta t}{\left(\rho_{v} \Delta h_{i}\right) R_{i}^{\prime} \theta_{i}} C_{q, i}\right)= \\
T_{L, i}^{t}+q_{a, i-1}^{t+1}\left(\frac{\lambda \rho_{a} \Delta t A_{q, i}}{\left(\rho_{v} \Delta h_{i}\right) R_{i}^{\prime} \theta_{i}}+\frac{C_{p}^{a i r} \rho_{a} \Delta t D_{T, i}}{\left(\rho_{v} \Delta h_{i}\right) R_{i} \theta_{i}}\right) \\
+T_{a, i-1}^{t+1}\left(\frac{C_{p}^{a i r} \rho_{a} \Delta t A_{T, i}}{\left(\rho_{v} \Delta h_{i}\right) R_{i} \theta_{i}}+\frac{\lambda \rho_{a} \Delta t D_{q, i}}{\left(\rho_{v} \Delta h_{i}\right) R_{i} \theta_{i}}\right)+\frac{\eta_{2} \Delta t}{\left(\rho_{v} \Delta h_{i}\right) \theta_{i}} \\
+\frac{\eta_{3} R_{S W, i}^{\text {down }} \Delta t}{\left(\rho_{v} \Delta h_{i}\right) \theta_{i}}+\frac{C_{p}^{a i r} \rho_{a} \Delta t}{\left(\rho_{v} \Delta h_{i}\right) R_{i} \theta_{i}} B_{T, i}+\frac{\lambda \rho_{a} \Delta t}{\left(\rho_{v} \Delta h_{i}\right) R_{i}^{\prime} \theta_{i}} B_{q, i}-\frac{\lambda \rho_{a} \Delta t}{\left(\rho_{v} \Delta h_{i}\right) R_{i}^{\prime} \theta_{i}} \beta_{i}
\end{gathered}
$$


So, to abbreviate (where $E_{i}, F_{i}$ and $G_{i}$ are known assumed constants for the level and timestep in question, (S3.10) can be written as:

iii) $T_{l e a f, i}^{t+1}=E_{i} q_{a, i-1}^{t+1}+F_{i} T_{a, i-1}^{t+1}+G_{i}$

so we define the coefficients as:

$$
\begin{aligned}
& E_{i}=\left(\frac{\Delta t A_{q, i} \lambda \rho_{a}}{\left(\rho_{v} \Delta h_{i}\right) R_{i}^{\prime} \theta_{i}}+\frac{\Delta t D_{T, i} C_{p}^{a i r} \rho_{a}}{\left(\rho_{v} \Delta h_{i}\right) R_{i} \theta_{i}}\right) / \\
& \left(1+\frac{\Delta t \alpha \lambda \rho_{a}}{\left(\rho_{v} \Delta h_{i}\right) R_{i}^{\prime} \theta_{i}}+\frac{\Delta t C_{p}^{a i r} \rho_{a}}{\left(\rho_{v} \Delta h_{i}\right) \theta_{i} R_{i}}-\frac{\eta_{1} \Delta t}{\left(\rho_{v} \Delta h_{i}\right) \theta_{i}}-\frac{\Delta t \lambda \rho_{a}}{\left(\rho_{v} \Delta h_{i}\right) R_{i}^{\prime} \theta_{i}} C_{q, i}-\frac{\Delta t C_{p}^{a i r} \rho_{a}}{\left(\rho_{v} \Delta h_{i}\right) \theta_{i} R_{i}} C_{T, i}\right)
\end{aligned}
$$

$$
\begin{aligned}
& F_{i}=\left(\frac{\Delta t A_{T, i} C_{p}^{a i r} \rho_{a}}{\left(\rho_{v} \Delta h_{i}\right) R_{i} \theta_{i}}+\frac{\Delta t D_{q, i} \lambda \rho_{a}}{\left(\rho_{v} \Delta h_{i}\right) R_{i}^{\prime} \theta_{i}}\right) / \\
& \left(1+\frac{\Delta t \alpha \lambda \rho_{a}}{\left(\rho_{v} \Delta h_{i}\right) R_{i}^{\prime} \theta_{i}}+\frac{\Delta t C_{p}^{a i r} \rho_{a}}{\left(\rho_{v} \Delta h_{i}\right) \theta_{i} R_{i}}-\frac{\eta_{1} \Delta t}{\left(\rho_{v} \Delta h_{i}\right) \theta_{i}}-\frac{\Delta t \lambda \rho_{a}}{\left(\rho_{v} \Delta h_{i}\right) R_{i}^{\prime} \theta_{i}} C_{q, i}-\frac{\Delta t C_{p}^{a i r} \rho_{a}}{\left(\rho_{v} \Delta h_{i}\right) \theta_{i} R_{i}} C_{T, i}\right)
\end{aligned}
$$

$$
\begin{aligned}
G_{i}= & \left(T_{\text {leaf }, i}^{t}+\frac{\eta_{2} \Delta t}{\left(\rho_{v} \Delta h_{i}\right) \theta_{i}}+\frac{\eta_{3} R_{S W}^{\text {down }} \Delta t}{\left(\rho_{v} \Delta h_{i}\right) \theta_{i}}-\frac{\lambda \rho_{a} \Delta t \beta_{i}}{\left(\rho_{v} \Delta h_{i}\right) R_{i}^{\prime} \theta_{i}}\right. \\
& \left.+\frac{\Delta t C_{p}^{\text {air }} \rho_{a}}{\left(\rho_{v} \Delta h_{i}\right) R_{i} \theta_{i}} B_{T, i}+\frac{\Delta t \lambda \rho_{a}}{\left(\rho_{v} \Delta h_{i}\right) R_{i}^{\prime} \theta_{i}} B_{q, i}\right) \\
/(1+ & \left.\frac{\Delta t \alpha \lambda \rho_{a}}{\left(\rho_{v} \Delta h_{i}\right) R_{i}^{\prime} \theta_{i}}+\frac{\Delta t C_{p}^{a i r} \rho_{a}}{\left(\rho_{v} \Delta h_{i}\right) \theta_{i} R_{i}}-\frac{\eta_{1} \Delta t}{\left(\rho_{v} \Delta h_{i}\right) \theta_{i}}-\frac{\Delta t \lambda \rho_{a}}{\left(\rho_{v} \Delta h_{i}\right) R_{i}^{\prime} \theta_{i}} C_{q, i}-\frac{\Delta t C_{p}^{a i r} \rho_{a}}{\left(\rho_{v} \Delta h_{i}\right) \theta_{i} R_{i}} C_{T, i}\right)
\end{aligned}
$$

\section{S3.6 Solving latent and sensible heat flux equations between layers by induction}

To prove by induction, we must express $T_{a, i}^{t+1}$ and $q_{a, i}^{t+1}$ in terms that are identical to (S3.5) and (S3.6) We first seek to eliminate $T_{a, i+1}^{t+1}$ from b) and c) We first 
substitute the assumed expressions for temperature and humidity in the layer above or, that is to say, (equations (S3.7) and (S3.8) here) We substitute for $T_{a, i+1}^{t+1}$ in b), to eliminate that term:

b) $\begin{aligned} \frac{T_{a, i}^{t+1}-T_{a, i}^{t}}{\Delta t} & =k_{i} \frac{A_{T, i+1} T_{a, i}^{t+1}+B_{T, i+1}+C_{T, i+1} T_{\text {leaf }, i+1}^{t+1}+D_{T, i+1} q_{a, i}^{t+1}}{\Delta z_{i} \Delta h_{i}} \\ & -\frac{k_{i} T_{a, i}^{t+1}}{\Delta z_{i} \Delta h_{i}}-\frac{k_{i-1} T_{a, i}^{t+1}}{\Delta z_{i-1} \Delta h_{i}}+\frac{k_{i-1} T_{a, i-1}^{t+1}}{\Delta z_{i-1} \Delta h_{i}}+\frac{T_{l e a f, i}^{t+1}}{\Delta h_{i} R_{i}}-\frac{T_{a, i}^{t+1}}{\Delta h_{i} R_{i}}\end{aligned}$

b) $T_{a, i}^{t+1}\left(1-\Delta t\left(A_{T, i+1} \frac{k_{i}}{\Delta z_{i} \Delta h_{i}}-\frac{k_{i}}{\Delta z_{i} \Delta h_{i}}-\frac{k_{i-1}}{\Delta z_{i-1} \Delta h_{i}}-\frac{1}{\Delta h_{i} R_{i}}\right)\right)=T_{a, i}^{t}+\frac{B_{T, i+1} k_{i} \Delta t}{\Delta z_{i} \Delta h_{i}}$

$$
+T_{a, i-1}^{t+1}\left(\frac{k_{i-1}}{\Delta z_{i-1} \Delta h_{i}}\right) \Delta t+q_{a, i}^{t+1}\left(\frac{k_{i} D_{T, i+1}}{\Delta z_{i} \Delta h_{i}}\right) \Delta t+T_{\text {leaf }, i}^{t+1}\left(\frac{\Delta t}{\Delta h_{i} R_{i}}\right)+T_{\text {leaf }, i+1}^{t+1}\left(\frac{k_{i} C_{T, i+1}}{\Delta z_{i} \Delta h_{i}}\right)
$$

Similarly, we substitute for $q_{a, i+1}^{t+1}$ in c), in order to eliminate that term:

$\frac{q_{a, i}^{t+1}-q_{a, i}^{t}}{\Delta t}=k_{i} \frac{\left(A_{q, i+1} q_{a, i}^{t+1}+B_{q, i+1}+C_{q, i+1} T_{l e a f, i+1}^{t+1}+D_{q, i+1} T_{a, i}^{t+1}\right)}{\Delta z_{i} \Delta h_{i}}$
$-\frac{k_{i} q_{a, i}^{t+1}}{\Delta z_{i} \Delta h_{i}}-\frac{k_{i-1} q_{a, i}^{t+1}}{\Delta z_{i-1} \Delta h_{i}}+\frac{k_{i-1} q_{a, i-1}^{t+1}}{\Delta z_{i-1} \Delta h_{i}}+\frac{\alpha_{i} T_{l e a f, i}^{t+1}+\beta_{i}-q_{a, i}^{t+1}}{\Delta h_{i} R_{i}^{\prime}}$

c) $q_{a, i}^{t+1}\left(1-\Delta t\left(A_{q, i+1} \frac{k_{i}}{\Delta z_{i} \Delta h_{i}}-\frac{k_{i}}{\Delta z_{i} \Delta h_{i}}-\frac{k_{i-1}}{\Delta z_{i-1} \Delta h_{i}}-\frac{1}{\Delta h_{i} R_{i}^{\prime}}\right)\right)=$

$$
\begin{aligned}
& q_{a, i}^{t}+\left(\frac{B_{q, i+1} k_{i} \Delta t}{\Delta z_{i} \Delta h_{i}}+\frac{\beta \Delta t}{\Delta h_{i} R_{i}^{\prime}}\right)+q_{a, i-1}^{t+1}\left(\frac{k_{i-1}}{\Delta z_{i-1} \Delta h_{i}} \Delta t\right) \\
& T_{a, i}^{t+1}\left(\frac{k_{i} D_{q, i+1}}{\Delta z_{i} \Delta h_{i}}\right) \Delta t+\left(T_{\text {leaf }, i+1}^{t+1}\right)\left(\frac{k_{i}}{\Delta z_{i} \Delta h_{i}}\right) C_{q, i+1} \Delta t+T_{\text {leaf }, i}^{t+1}\left(\frac{\alpha_{i}}{\Delta h_{i} R_{i}^{\prime}}\right) \Delta t
\end{aligned}
$$

Now, we substitute expression iii) for the leaf temperature in the layer above (S3.11). This step is in order to eliminate the term $T_{L, i+1}^{t+1}$ from both expressions: 
b) $T_{a, i}^{t+1}\left(1-\Delta t\left(A_{T, i+1} \frac{k_{i}}{\Delta z_{i} \Delta h_{i}}-\frac{k_{i}}{\Delta z_{i} \Delta h_{i}}-\frac{k_{i-1}}{\Delta z_{i-1} \Delta h_{i}}-\frac{1}{\Delta h_{i} R_{i}}\right)\right)=$

$$
\begin{aligned}
T_{a, i}^{t}+\frac{B_{T, i+1} k_{i} \Delta t}{\Delta z_{i} \Delta h_{i}}+ & T_{a, i-1}^{t+1}\left(\frac{k_{i-1}}{\Delta z_{i-1} \Delta h_{i}}\right) \Delta t+q_{a, i}^{t+1}\left(\frac{k_{i} D_{T, i+1}}{\Delta z_{i} \Delta h_{i}}\right) \Delta t+T_{\text {leaf }, i}^{t+1}\left(\frac{\Delta t}{\Delta h_{i} R_{i}}\right) \\
& +\left(E_{i+1} q_{a, i}^{t+1}+F_{i+1} T_{a, i}^{t+1}+G_{i+1}\right)\left(\frac{k_{i} C_{T, i+1}}{\Delta z_{i} \Delta h_{i}}\right) \Delta t \quad(\mathrm{~S} 3.19)
\end{aligned}
$$

c) $q_{a, i}^{t+1}\left(1-\Delta t\left(A_{q, i+1} \frac{k_{i}}{\Delta z_{i} \Delta h_{i}}-\frac{k_{i}}{\Delta z_{i} \Delta h_{i}}-\frac{k_{i-1}}{\Delta z_{i-1} \Delta h_{i}}-\frac{1}{\Delta h_{i} R_{i}^{\prime}}\right)\right)=$

$$
\begin{array}{r}
q_{a, i}^{t}+\left(\frac{B_{q, i+1} k_{i} \Delta t}{\Delta z_{i} \Delta h_{i}}+\frac{\beta \Delta t}{\Delta h_{i} R_{i}^{\prime}}\right)+q_{a, i-1}^{t+1}\left(\frac{k_{i-1}}{\Delta z_{i-1} \Delta h_{i}} \Delta t\right) \\
T_{a, i}^{t+1}\left(\frac{k_{i} D_{q, i+1}}{\Delta z_{i} \Delta h_{i}}\right) \Delta t+\left(E_{i+1} q_{a, i}^{t+1}+F_{i+1} T_{a, i}^{t+1}+G_{i+1}\right)\left(\frac{k_{i}}{\Delta z_{i} \Delta h_{i}}\right) C_{q, i+1} \Delta t \\
+T_{\text {leaf }, i}^{t+1}\left(\frac{\alpha}{\Delta h_{i} R_{i}^{\prime}}\right) \Delta t
\end{array}
$$

We now abbreviate equation a) as:

$$
\text { b) } \quad T_{a, i}^{t+1} X_{1, i}=X_{2, i}+X_{3, i} T_{a, i-1}^{t+1}+X_{4, i} q_{a, i}^{t+1}+X_{5, i} T_{l e a f, i}^{t+1}
$$

and abbreviate equation b) as:

$$
\text { c) } \quad q_{a, i}^{t+1} Y_{1, i}=Y_{2, i}+Y_{3, i} q_{a, i-1}^{t+1}+Y_{4, i} T_{a, i}^{t+1}+Y_{5, i} T_{l e a f, i}^{t+1}
$$

where:

$$
\begin{aligned}
& X_{1, i}=1-\Delta t\left(A_{T, i+1} \frac{k_{i}}{\Delta z_{i} \Delta h_{i}}-\frac{k_{i}}{\Delta z_{i} \Delta h_{i}}-\frac{k_{i-1}}{\Delta z_{i-1} \Delta h_{i}}-\frac{1}{\Delta h_{i} R_{i}}\right) \\
& \quad-F_{i+1}\left(\frac{k_{i} C_{T, i+1}}{\Delta z_{i} \Delta h_{i}}\right) \Delta t \\
& X_{2, i}=T_{a, i}^{t}+\frac{B_{T, i+1} k_{i} \Delta t}{\Delta z_{i} \Delta h_{i}}+G_{i+1}\left(\frac{k_{i} C_{T, i+1}}{\Delta z_{i} \Delta h_{i}}\right) \Delta t \\
& X_{3, i}=\left(\frac{k_{i-1}}{\Delta z_{i-1} \Delta h_{i}}\right) \Delta t
\end{aligned}
$$




$$
\begin{aligned}
X_{4, i} & =\left(\frac{k_{i} D_{T, i+1}}{\Delta z_{i} \Delta h_{i}}\right) \Delta t+E_{i+1}\left(\frac{k_{i} C_{T, i+1}}{\Delta z_{i} \Delta h_{i}}\right) \Delta t \\
X_{5, i} & =\left(\frac{\Delta t}{\Delta h_{i} R_{i}}\right) \\
Y_{1, i} & =1-\Delta t\left(A_{q, i+1} \frac{k_{i}}{\Delta z_{i} \Delta h_{i}}-\frac{k_{i}}{\Delta z_{i} \Delta h_{i}}-\frac{k_{i-1}}{\Delta z_{i-1} \Delta h_{i}}-\frac{1}{\Delta h_{i} R_{i}^{\prime}}\right) \\
Y_{2, i} & =q_{a, i}^{t}+\left(\frac{B_{q, i+1} k_{i} \Delta t}{\Delta z_{i} \Delta h_{i}}+\frac{\beta_{i} \Delta t}{\Delta h_{i} R_{i}^{\prime}}\right)+G_{i+1}\left(\frac{k_{i}}{\Delta z_{i} \Delta h_{i}}\right) C_{q, i+1} \Delta t \\
Y_{3, i} & =\left(\frac{k_{i-1}}{\Delta z_{i-1} \Delta h_{i}} \Delta t\right) \\
Y_{4, i} & =\left(\frac{k_{i} D_{q, i+1}}{\Delta z_{i} \Delta h_{i}}\right) \Delta t+C_{q, i+1} \Delta t \\
Y_{5, i} & =\left(\frac{\alpha_{i}}{\Delta h_{i} R_{i}^{\prime}}\right) \Delta t
\end{aligned}
$$

We then cross-substitute for $q_{a, i}^{t+1}$ from c) to b), to eliminate that term:

b) $T_{a, i}^{t+1} X_{1, i}=X_{2, i}+X_{3, i} T_{a, i-1}^{t+1}+X_{4, i}\left(\frac{Y_{2, i}}{Y_{1, i}}+\frac{Y_{3, i}}{Y_{1, i}} q_{a, i-1}^{t+1}+\frac{Y_{4, i}}{Y_{1, i}} T_{a, i}^{t+1}+\frac{Y_{5, i}}{Y_{1, i}} T_{l e a f, i}^{t+1}\right)$

$$
+X_{5, i} T_{l e a f, i}^{t+1}
$$

b) $T_{a, i}^{t+1}\left(X_{1, i}-X_{4, i} \frac{Y_{4, i}}{Y_{1, i}}\right)=T_{a, i-1}^{t+1} X_{3, i}+\left(X_{2, i}+X_{4, i} \frac{Y_{2, i}}{Y_{1, i}}\right)$

$$
+T_{L, i}^{t+1}\left(X_{4, i} \frac{Y_{5, i}}{Y_{1, i}}+X_{5, i}\right)+q_{a, i-1}^{t+1}\left(X_{4, i} \frac{Y_{3, i}}{Y_{1, i}}\right)
$$

similarly, we cross-substitute for $T_{a, i}^{t+1}$ from b) to a), to eliminate that term:

$$
\begin{array}{r}
q_{a, i}^{t+1} Y_{1, i}=Y_{2, i}+Y_{3, i} q_{a, i-1}^{t+1}+Y_{4, i}\left(\frac{X_{2, i}}{X_{1, i}}+\frac{X_{3, i}}{X_{1, i}} T_{a, i-1}^{t+1}+\frac{X_{4, i}}{X_{1, i}} q_{a, i}^{t+1}+\frac{X_{5, i}}{X_{1, i}} T_{\text {leaf }, i}^{t+1}\right) \\
+Y_{5, i} T_{\text {leaf }, i}^{t+1}
\end{array}
$$




$$
\text { c) } \begin{aligned}
q_{a, i}^{t+1}\left(Y_{1, i}-Y_{4, i} \frac{X_{4, i}}{X_{1, i}}\right) & =q_{a, i-1}^{t+1} Y_{3, i}+\left(Y_{2, i}+Y_{4, i} \frac{X_{2, i}}{X_{1, i}}\right) \\
+ & T_{\text {leaf }, i}^{t+1}\left(Y_{4, i} \frac{X_{5, i}}{X_{1, i}}+Y_{5, i}\right)+T_{a, i-1}^{t+1}\left(Y_{4, i} \frac{X_{3, i}}{X_{1, i}}\right)
\end{aligned}
$$

So this demonstrates the expressions b) and c) can be described in terms of the respective original substitutions (S3.5) and (S3.6). The respective coefficients from (S3.5) and (S3.6) may be described as:

$$
\begin{gathered}
A_{T, i}=\frac{X_{3, i}}{X_{1, i}-X_{4, i}\left(\frac{Y_{4, i}}{Y_{1, i}}\right)} \\
B_{T, i}=\frac{X_{2, i}+X_{4, i}\left(\frac{Y_{2, i}}{Y_{1, i}}\right)}{X_{1, i}-X_{4, i}\left(\frac{Y_{4, i}}{Y_{1, i}}\right)} \\
C_{T, i}=\frac{\left(X_{4, i}\left(\frac{Y_{5, i}}{Y_{1, i}}\right)+X_{5, i}\right)}{X_{1, i}-X_{4, i}\left(\frac{Y_{4, i}}{Y_{1, i}}\right)} \\
D_{T, i}=\frac{X_{4, i}\left(\frac{Y_{3, i}}{Y_{1, i}}\right)}{X_{1, i}-X_{4, i}\left(\frac{Y_{4, i}}{Y_{1, i}}\right)}
\end{gathered}
$$

and:

$$
\begin{aligned}
A_{q, i} & =\frac{Y_{3, i}}{Y_{1, i}-Y_{4, i}\left(\frac{X_{4, i}}{X_{1, i}}\right)} \\
B_{q, i} & =\frac{Y_{2, i}+Y_{4, i}\left(\frac{X_{2, i}}{X_{1, i}}\right)}{Y_{1, i}-Y_{4, i}\left(\frac{X_{4, i}}{X_{1, i}}\right)} \\
C_{q, i} & =\frac{\left(Y_{4, i}\left(\frac{X_{5, i}}{X_{1, i}}\right)+Y_{5, i}\right)}{Y_{1, i}-Y_{4, i}\left(\frac{X_{4, i}}{X_{1, i}}\right)} \\
D_{q, i} & =\frac{Y_{4, i}\left(\frac{X_{3, i}}{X_{1, i}}\right)}{Y_{1, i}-Y_{4, i}\left(\frac{X_{4, i}}{X_{1, i}}\right)}
\end{aligned}
$$

Now, all of the coefficients $X_{1, i}, X_{2, i}, X_{3, i}, X_{4, i}, X_{5, i}, Y_{1, i}, Y_{2, i}, Y_{3, i}, Y_{4, i}$ and $Y_{5, i}$ and, in turn, the coefficients $A_{T, i}, B_{T, i}, C_{T, i}, D_{T, i}, A_{q, i}, B_{q, i}, C_{q, i}$ and $D_{q, i}$ can be described 
in terms of the coefficients from the level above and the potentials (i.e. $\mathrm{T}$ and $\mathrm{q}$ ) at the previous timestep.

So we have a set of coefficients that may be determined for each time-step, and we have the means to determine $T_{\text {surf }}$ (and $q_{\text {surf }}$ via the saturation assumption). We thus have a process to calculate the temperature and humidity profiles for each timestep by systematically calculating each of the coefficients from the top of the column (the 'downwards sweep') then calculating the 'initial value' (the surface temperature and humidity) and finally calculating each $T_{a}, q_{a}$ and $T_{l e a f}$ by working up the column (the 'upwards sweep').

The term $T_{\text {leaf }, i+1}^{t+1}$ can also be described in terms of the variables at the level below by using equation iii) and its terms $E_{i}, F_{i}$ and $G_{i}$. We can therefore describe the changes in the canopy between the present timestep $t$ and the next timestep $t+1$ by 'working down' the column from the interaction with the LMDZ atmospheric model to determine the coefficients $A_{T}, B_{T}, C_{T}$ etc. and then 'working up' the column to determine the potentials $\mathrm{T}$ and $\mathrm{q}$. 


\section{S3.7 Alternative derivation}

A more direct method of derivation is to assume solutions of the form:

$$
{\overrightarrow{u_{i}}}^{t+1}=\boldsymbol{E}_{\boldsymbol{i}}^{\prime}{\overrightarrow{u_{i+1}}}^{t+1}+\boldsymbol{F}_{\boldsymbol{i}}^{\prime}
$$

where we define the vector $\vec{u}_{i}^{t+1}$ such that:

$$
{\overrightarrow{u_{i}}}^{t+1}=\left(\begin{array}{c}
T_{a, i}^{t+1} \\
q_{a, i}^{t+1}
\end{array}\right)
$$

Now, the original expression for the leaf temperature is:

$$
\begin{array}{r}
\theta_{i} \rho_{v} \frac{\left(T_{\text {leaf }, i}^{t+1}-T_{\text {leaf }, i}^{t}\right)}{\Delta t}=\left(\frac{1}{\Delta h_{i}}\right)\left(-C_{p}^{a i r} \rho_{a} \frac{\left(T_{\text {leaf }, i}^{t+1}-T_{a, i}^{t+1}\right)}{R_{i}}\right. \\
\left.-\lambda \rho_{a} \frac{\left(\alpha_{i} T_{\text {leaf }, i}^{t+1}+\beta_{i}-q_{a, i}^{t+1}\right)}{R_{i}^{\prime}}+\eta_{1} T_{\text {leaf }, i}^{t+1}+\eta_{2}+\eta_{3} R_{S W}^{d o w n}\right)
\end{array}
$$

which we can abbreviate (with a change of variable label here, to reduce confusion) as:

a) $T_{L, i}^{t+1}=X_{i} q_{a, i}^{t+1}+Y_{i} T_{a, i}^{t+1}+Z_{i}$

The original expression for the temperature column is:

$$
\text { b) } \begin{aligned}
\frac{T_{a, i}^{t+1}-T_{a, i}^{t}}{\Delta t}=k_{i}\left(\frac{\left(T_{a, i+1}^{t+1}-T_{a, i}^{t+1}\right)}{\Delta z_{i} \Delta h_{i}}\right)-k_{i-1} & \left(\frac{\left(T_{a, i}^{t+1}-T_{a, i-1}^{t+1}\right)}{\Delta z_{i-1} \Delta h_{i}}\right) \\
+ & \left(\frac{1}{\Delta h_{i}}\right) \frac{\left(T_{\text {leaf }, i}^{t+1}-T_{a, i}^{t+1}\right)}{R_{i}}
\end{aligned}
$$

The original expression for the specific humidity column is:

$$
\text { c) } \begin{aligned}
\frac{q_{a, i}^{t+1}-q_{a, i}^{t}}{\Delta t}=k_{i}\left(\frac{\left(q_{a, i+1}^{t+1}-q_{a, i}^{t+1}\right)}{\Delta z_{i} \Delta h_{i}}\right) & -k_{i-1}\left(\frac{\left(q_{a, i}^{t+1}-q_{a, i-1}^{t+1}\right)}{\Delta z_{i-1} \Delta h_{i}}\right) \\
& +\left(\frac{1}{\Delta h_{i}}\right) \frac{\left(\alpha_{i} T_{\text {leaf }, i}^{t+1}+\beta_{i}-q_{a, i}^{t+1}\right)}{R_{i}^{\prime}}
\end{aligned}
$$

which we abbreviate to the form of Richtmyer and Morton (1967), equation 11.7, substituting for $T_{L, i}^{t+1}$ from a), above:

$$
\text { b) }-\boldsymbol{A}_{\boldsymbol{i}}^{\prime}{\overrightarrow{u_{i+1}}}^{t+1}+\boldsymbol{B}_{\boldsymbol{i}}^{\prime}{\overrightarrow{u_{i}}}^{t+1}-\boldsymbol{C}_{\boldsymbol{i}}^{\prime}{\overrightarrow{u_{i-1}}}^{t+1}=\boldsymbol{D}_{\boldsymbol{i}}^{\prime}
$$


Now, directly from Richtmyer and Morton (1967), we can substitute for $\vec{u}_{i-1}^{t+1}=$ $\boldsymbol{E}_{\boldsymbol{i}-\mathbf{1}}^{\prime} \vec{u}_{i}^{t+1}+\boldsymbol{F}_{\boldsymbol{i}-\mathbf{1}}^{\prime}$. This results in a pair of expressions of the form:

$$
\left.\vec{u}_{a, i}^{t+1}=\left(B_{i}^{\prime}-C_{i}^{\prime} E_{i-1}^{\prime}\right)^{-1} A_{i}^{\prime} \vec{u}_{a, i+1}^{t+1}+B_{i}^{\prime}-C_{i}^{\prime} E_{i-1}^{\prime}\right)^{-1} D_{i}^{\prime}+C_{i}^{\prime} F_{i-1}^{\prime}(
$$

So we can describe the variables for (S3.45) as:

$$
\begin{aligned}
& E_{i}^{\prime}=\left(B_{i}^{\prime}-C_{i}^{\prime} E_{i-1}^{\prime}\right)^{-1} A_{i}^{\prime} \\
& F_{i}^{\prime}=\left(B_{i}^{\prime}-C_{i}^{\prime} E_{i-1}^{\prime}\right)^{-1}\left(D_{i}^{\prime}+C_{i}^{\prime} F_{i-1}^{\prime}\right)
\end{aligned}
$$

Now $A_{i}^{\prime}, B_{i}^{\prime}, C_{i}^{\prime}$ and $D_{i}^{\prime}$ may be described from substitution of the equations (S3.47), (S3.48) and (S3.49).

We thus have a set of two equations to solve simultaneously, which is possible starting from the upper boundary conditions, as laid out in section S4.1. However, we still need to describe $\boldsymbol{E}_{\boldsymbol{i}}^{\prime}$ and $\boldsymbol{F}_{\boldsymbol{i}}^{\prime}$ which can be achieved by rearranging equations (S4.3), (S4.4), (S4.7), (S4.30) and (S4.31), so that we have two expressions of the form:

$$
\vec{u}_{\text {surf }}^{t+1}=\boldsymbol{E}_{\mathbf{0}}^{\prime} u_{1}^{t+1}+\boldsymbol{F}_{\mathbf{0}}^{\prime}
$$

So if we can describe $\boldsymbol{E}_{\mathbf{0}}^{\prime}$ and $\boldsymbol{F}_{\mathbf{0}}^{\prime}$ from use of (S3.52) and (S3.53), we can then derive the value of these variables working up the column. 
Table 1: Input coefficients at the top layer of the model, where $A_{T, n}, B_{T, n} \ldots$ etc are the respective coefficients at the top of the surface model and $A_{T, \text { atmos }}, B_{T, \text { atmos }}$ are the coefficients at the lowest level of the atmospheric model.

\begin{tabular}{|l|l|}
\hline stand-alone model & coupled model \\
\hline$A_{T, n}=0$ & $A_{T, n}=A_{T, \text { atmos }}$ \\
$B_{T, n}=B_{T, \text { input }}$ & $B_{T, n}=B_{T, \text { atmos }}$ \\
$C_{T, n}=0$ & $C_{T, n}=0$ \\
$D_{T, n}=0$ & $D_{T, n}=0$ \\
\hline$A_{q, n}=0$ & $A_{q, n}=A_{q, \text { atmos }}$ \\
$B_{q, n}=B_{q, \text { input }}$ & $B_{q, n}=B_{q, \text { atmos }}$ \\
$C_{q, n}=0$ & $C_{q, n}=0$ \\
$D_{q, n}=0$ & $D_{q, n}=0$ \\
\hline
\end{tabular}

\section{S4 The boundary conditions}

\section{S4.1 The upper boundary conditions}

In stand-alone simulations, the top level variables $A_{T, n}, C_{T, n}, D_{T, n}$ and $A_{q, n}, C_{q, n}, D_{q, n}$, are set to zero and $B_{T, n}$ and $B_{q, n}$ set to the input temperature and specific humidity respectively for the relevant time step (as in Best et al. (2004)) In coupled simulations, $A_{T, n}, B_{T, n}$ and $A_{q, n}, B_{q, n}$ are taken from the respective values at lowest level of the atmospheric model. Table 1 summarises the boundary conditions for both the coupled and un-coupled simulations. 


\section{S4.2 The lower boundary condition}

We need to solve the lowest level transport equations separately:

$$
\begin{array}{r}
\text { b) } \frac{T_{a, 1}^{t+1}-T_{a, 1}^{t}}{\Delta t}=k_{1}\left(\frac{T_{a, 2}^{t+1}-T_{a, 1}^{t+1}}{\Delta z_{1} \Delta h_{1}}\right)-\left(\frac{1}{\rho_{a} C_{p}^{a i r}}\right) \frac{\phi_{H}^{t+1}}{\Delta h_{1}}+\left(\frac{1}{\Delta h_{1}}\right)\left(\frac{T_{L, 1}^{t+1}-T_{a, 1}^{t+1}}{R_{1}}\right) \\
\text { c) } \frac{q_{a, 1}^{t+1}-q_{a, 1}^{t}}{\Delta t}=k_{1}\left(\frac{q_{a, 2}^{t+1}-q_{a, 1}^{t+1}}{\Delta z_{1} \Delta h_{1}}\right)-\left(\frac{1}{\rho_{a} \lambda}\right) \frac{\phi_{\lambda E}^{t+1}}{\Delta h_{1}}+\left(\frac{1}{\Delta h_{1}}\right)\left(\frac{\alpha_{i} T_{L, 1}^{t+1}+\beta_{1}-q_{a, 1}^{t+1}}{R_{1}^{\prime}}\right)
\end{array}
$$

We substitute to the above to eliminate $T_{a, 2}^{t+1}$ from b) and $q_{a, 2}^{t+1}$ from c):

$$
T_{a, 1}^{t+1}=A_{T, 1} \phi_{H}^{t+1}+B_{T, 1}+C_{T, 1} T_{L, 1}^{t+1}+D_{T, 1} \phi_{\lambda E}^{t+1}
$$

and:

$$
q_{a, 1}^{t+1}=A_{q, 1} \phi_{\lambda E}^{t+1}+B_{q, 1}+C_{q, 1} T_{L, 1}^{t+1}+D_{q, 1} \phi_{H}^{t+1}
$$

Now for the leaf at level 1, just above the ground level:

a) $\quad T_{\text {leaf }, 1}^{t+1}-T_{\text {leaf }, 1}^{t}=\frac{\lambda \rho_{a} \Delta t \beta_{1}}{\left(\rho_{v} \Delta h_{1}\right) R_{1}^{\prime} \theta_{1}}+\frac{\eta_{3} R_{S W}^{\text {down }} \Delta t}{\left(\rho_{v} \Delta h_{1}\right) \theta_{1}}+\frac{\eta_{2} \Delta t}{\left(\rho_{v} \Delta h_{1}\right) \theta_{1}}$

$$
\begin{aligned}
& +T_{\text {leaf }, 1}^{t+1}\left(C_{p}^{a i r} \rho_{a} \frac{\Delta t}{\left(\rho_{v} \Delta h_{1}\right) R_{1} \theta_{1}}+\lambda \rho_{a} \frac{\Delta t \alpha}{\left(\rho_{v} \Delta h_{1}\right) R_{1}^{\prime} \theta_{1}}+\frac{\eta_{1} \Delta t}{\left(\rho_{v} \Delta h_{1}\right) \theta_{1}}\right) \\
& -T_{a, 1}^{t+1} C_{p}^{a i r} \rho_{a}\left(\frac{\Delta t}{R_{1} \theta_{1}\left(\rho_{v} \Delta h_{1}\right)}\right)-q_{a, 1}^{t+1} \lambda \rho_{a}\left(\frac{\Delta t}{\left(\rho_{v} \Delta h_{1}\right) R_{1}^{\prime} \theta_{1}}\right)
\end{aligned}
$$

and substitute for $T_{a, 1}^{t+1}$ and $q_{a, 1}^{t+1}$ :

a) $\quad T_{\text {leaf }, 1}^{t+1}-T_{\text {leaf }, 1}^{t}=\frac{\lambda \rho_{a} \Delta t \beta_{i}}{\left(\rho_{v} \Delta h_{1}\right) R_{1}^{\prime} \theta_{1}}+\frac{\eta_{3} R_{S W}^{\text {down }} \Delta t}{\left(\rho_{v} \Delta h_{1}\right) \theta_{1}}+\frac{\eta_{2} \Delta t}{\left(\rho_{v} \Delta h_{1}\right) \theta_{1}}$

$$
\begin{aligned}
& +T_{\text {leaf }, 1}^{t+1}\left(C_{p}^{a i r} \rho_{a} \frac{\Delta t}{\left(\rho_{v} \Delta h_{1}\right) R_{1} \theta_{1}}+\lambda \rho_{a} \frac{\Delta t \alpha}{\left(\rho_{v} \Delta h_{1}\right) R_{1}^{\prime} \theta_{1}}+\frac{\eta_{1} \Delta t}{\left(\rho_{v} \Delta h_{1}\right) \theta_{1}}\right) \\
& -\frac{C_{p}^{a i r} \rho_{a} \Delta t}{R_{1} \theta_{1}\left(\rho_{v} \Delta h_{1}\right)}\left(A_{T, 1} \phi_{H}^{t+1}+B_{T, 1}+C_{T, 1} T_{\text {leaf }, 1}^{t+1}+D_{T, 1} \phi_{\lambda E}^{t+1}\right) \\
& -\frac{\lambda \rho_{a} \Delta t}{R_{1}^{\prime} \theta_{1}\left(\rho_{v} \Delta h_{1}\right)}\left(A_{q, 1} \phi_{\lambda E}^{t+1}+B_{q, 1}+C_{q, 1} T_{\text {leaf }, 1}^{t+1}+D_{q, 1} \phi_{H}^{t+1}\right)
\end{aligned}
$$


In a similar approach to the previous section, this should be reduced to the form:

$$
T_{l e a f, 1}^{t+1}=E_{1} \phi_{\lambda E}^{t+1}+F_{1} \phi_{H}^{t+1}+G_{1}
$$

and the expression re-arranged to isolate the factors $E_{1}, F_{1}$ and $G_{1}$ :

$$
\begin{array}{r}
T_{\text {leaf }, 1}^{t+1}\left(1-\left(\frac{C_{p}^{a i r} \rho_{a} \Delta t}{\left(\rho_{v} \Delta h_{1}\right) R_{1} \theta_{1}}+\frac{\lambda \rho_{a} \Delta t \alpha}{\left(\rho_{v} \Delta h_{1}\right) R_{1}^{\prime} \theta_{1}}+\frac{\eta_{2} \Delta t}{\left(\rho_{v} \Delta h_{1}\right) \theta_{1}}\right)+\frac{C_{T, 1} C_{p}^{a i r} \rho_{a} \Delta t}{R_{1} \theta_{1}\left(\rho_{v} \Delta h_{1}\right)}\right. \\
\left.+\frac{C_{q, 1} \lambda \rho_{a} \Delta t}{\left(\rho_{v} \Delta h_{1}\right) R_{1}^{\prime} \theta_{1}}\right)=T_{\text {leaf }, 1}^{t}+\phi_{\lambda E}^{t+1}\left(-\frac{C_{p}^{a i r} \rho_{a} \Delta t}{R_{1} \theta_{1}\left(\rho_{v} \Delta h_{1}\right)} D_{T, 1}-\frac{\lambda \rho_{a} \Delta t}{\left(\rho_{v} \Delta h_{1}\right) R_{1}^{\prime} \theta_{1}} A_{q, 1}\right) \\
+\phi_{H}^{t+1}\left(-\frac{C_{p}^{a i r} \rho_{a} \Delta t}{R_{1} \theta_{1}\left(\rho_{v} \Delta h_{1}\right)} A_{T, 1}-\frac{\lambda r h o_{a} \Delta t}{\left(\rho_{v} \Delta h_{1}\right) R_{1}^{\prime} \theta_{1}} D_{q, 1}\right) \\
+\left(\frac{\lambda \rho_{a} \Delta t \beta_{i}}{\left(\rho_{v} \Delta h_{1}\right) R_{1}^{\prime} \theta_{1}}+\frac{\eta_{3} R_{S W}^{\text {down }} \Delta t}{\left(\rho_{v} \Delta h_{1}\right) \theta_{1}}\right. \\
\left.+\frac{\eta_{2} \Delta t}{\left(\rho_{v} \Delta h_{1}\right) \theta_{1}}-\frac{B_{T, 1} C_{p}^{a i r} \rho_{a} \Delta t}{R_{1} \theta_{1}\left(\rho_{v} \Delta h_{1}\right)}-\frac{B_{q, 1} \lambda \rho_{a} \Delta t}{\left(\rho_{v} \Delta h_{1}\right) R_{1}^{\prime} \theta_{1}}\right)
\end{array}
$$

Now, substituting for $T_{a, 2}^{t+1}$ in expression b):

b) $\frac{T_{a, 1}^{t+1}-T_{a, 1}^{t}}{\Delta t}=$

$$
\begin{array}{r}
k_{1} \frac{\left(A_{T, 2} T_{a, 1}^{t+1}+B_{T, 2}+C_{T, 2}\left(E_{2} q_{a, 1}^{t+1}+F_{2} T_{a, 1}^{t+1}+G_{2}\right)+D_{T, 2} q_{a, 1}^{t+1}+T_{a, 1}^{t+1}\right)}{\Delta z_{1} \Delta h_{1}} \\
-\left(\frac{1}{\rho_{a} C_{p}^{\text {air }}}\right) \frac{\phi_{H}^{t+1}}{\Delta h_{1}}+\frac{T_{\text {leaf }, 1}^{t+1}-T_{a, 1}^{t+1}}{R_{1} \Delta h_{1}} \quad(\mathrm{~S} 4.9)
\end{array}
$$

b) $T_{a, 1}^{t+1}\left(\frac{1}{\Delta t}-\frac{k_{1} A_{T, 2}}{\Delta z_{1} \Delta h_{1}}-\frac{k_{1} C_{T, 2} F_{2}}{\Delta z_{1} \Delta h_{1}}+\frac{k_{1}}{\Delta z_{1} \Delta h_{1}}+\frac{1}{\Delta h_{1} R_{1}}\right)=$

$$
\begin{aligned}
& \quad \frac{T_{a, 1}^{t}}{\Delta t}+q_{a, 1}^{t+1}\left(\frac{k_{1} C_{T, 2} E_{2}+k_{1} D_{T, 2}}{\Delta z_{1} \Delta h_{1}}\right)+ \\
& T_{\text {leaf }, 1}^{t+1}\left(\frac{1}{\Delta h_{1} R_{1}}\right)+\left(\frac{k_{1} B_{T, 2}}{\Delta z_{1} \Delta h_{1}}+\frac{k_{1} C_{T, 2} G_{2}}{\Delta z_{1} \Delta h_{1}}\right)-\left(\frac{1}{\rho_{a} C_{p}^{a i r}}\right) \phi_{H}^{t+1}\left(\frac{1}{\Delta h_{1}}\right)
\end{aligned}
$$

and for $q_{a, 2}^{t+1}$ in expression c): 
c) $\frac{q_{a, 1}^{t+1}-q_{a, 1}^{t}}{\Delta t}=k_{1} \frac{\left(A_{q, 2} q_{a, 1}^{t+1}+B_{q, 2}+C_{q, 2} T_{l e a f, 2}^{t+1}+D_{q, 2} T_{a, 1}^{t+1}-q_{a, 1}^{t+1}\right)}{\Delta z_{1} \Delta h_{1}}=$

$$
-\left(\frac{1}{\rho_{a} \lambda}\right) \frac{\phi_{\lambda E}^{t+1}}{\Delta h_{1}}+\frac{1}{\Delta h_{1}} \frac{\alpha T_{\text {leaf }, 1}^{t+1}+\beta_{1}-q_{a, 1}^{t+1}}{R_{1}^{\prime}}
$$

c) $\frac{q_{a, 1}^{t+1}-q_{a, 1}^{t}}{\Delta t}=k_{1} \frac{\left(A_{q, 2} q_{a, 1}^{t+1}+B_{q, 2}+C_{q, 2}\left(E_{2} q_{a, 1}^{t+1}+F_{2} T_{a, 1}^{t+1}+G_{2}\right)+D_{q, 2} T_{a, 1}^{t+1}-q_{a, 1}^{t+1}\right)}{\Delta z_{1} \Delta h_{1}}$

$$
-\left(\frac{1}{\rho_{a} \lambda}\right) \frac{\phi_{\lambda E}^{t+1}}{\Delta h_{1}}+\frac{1}{\Delta h_{1}} \frac{\left(\alpha T_{l e a f, 1}^{t+1}+\beta_{1}-q_{a, 1}^{t+1}\right)}{R_{1}^{\prime}}
$$

c) $q_{a, 1}^{t+1}\left(\frac{1}{\Delta t}-\frac{k_{1} A_{q, 2}}{\Delta z_{1} \Delta h_{1}}-\frac{k_{1} C_{q, 2} E_{2}}{\Delta z_{1} \Delta h_{1}}+\frac{k_{1}}{\Delta z_{1} \Delta h_{1}}\right)=$

$$
\begin{aligned}
& \frac{q_{a, 1}^{t}}{\Delta t}+T_{a, 1}^{t+1}\left(\frac{C_{q, 2} F_{2}}{\Delta z_{1} \Delta h_{1}}+\frac{D_{q, 2}}{\Delta z_{1} \Delta h_{1}}\right)+T_{L, 1}^{t+1}\left(\frac{\alpha}{\Delta h_{1} R_{1}^{\prime}}\right) \\
& +\left(\frac{k_{1} B_{q, 2}}{\Delta z_{1} \Delta h_{1}}+\frac{C_{q, 2} G_{2}}{\Delta z_{1} \Delta h_{1}}+\frac{\beta_{1}}{\Delta h_{1} R_{1}^{\prime}}\right)-\phi_{\lambda E}^{t+1}\left(\frac{1}{\Delta h_{1}}\right)
\end{aligned}
$$

We now isolate the terms in (S4.8):

a) $T_{L, 1}^{t+1}=E_{1} \phi_{\lambda E}^{t+1}+F_{1} \phi_{H}^{t+1}+G_{1}$

so we have:

$$
\begin{aligned}
& E_{1}=\left(-\frac{C_{p}^{a i r} \rho_{a} \Delta t}{R_{1} \theta_{1}\left(\rho_{v} \Delta h_{1}\right)} D_{T, 1}-\frac{\lambda \rho_{a} \Delta t}{\left(\rho_{v} \Delta h_{1}\right) R_{1}^{\prime} \theta_{1}} A_{q, 1}\right) / \\
& \left(1-\left(\frac{C_{p}^{a i r} \rho_{a} \Delta t}{\left(\rho_{v} \Delta h_{1}\right) R_{1} \theta_{1}}+\frac{\lambda \rho_{a} \Delta t \alpha_{i}}{\left(\rho_{v} \Delta h_{1}\right) R_{1}^{\prime} \theta_{1}}+\frac{\eta_{1} \Delta t}{\left(\rho_{v} \Delta h_{1}\right) \theta_{1}}\right)+\frac{C_{T, 1} C_{p}^{a i r} \rho_{a} \Delta t}{R_{1} \theta_{1}\left(\rho_{v} \Delta h_{1}\right)}+\frac{C_{q, 1} \lambda \rho_{a} \Delta t}{\left(\rho_{v} \Delta h_{1}\right) R_{1}^{\prime} \theta_{1}}\right) \\
& F_{1}=\left(-\frac{C_{p}^{a i r} \rho_{a} \Delta t}{R_{1} \theta_{1}\left(\rho_{v} \Delta h_{1}\right)} A_{T, 1}-\frac{\lambda \rho_{a} \Delta t}{\left(\rho_{v} \Delta h_{1}\right) R_{1}^{\prime} \theta} D_{q, 1}\right) / \\
& \left(1-\left(\frac{C_{p}^{a i r} \rho_{a} \Delta t}{\left(\rho_{v} \Delta h_{1}\right) R_{1} \theta_{1}}+\frac{\lambda \rho_{a} \Delta t \alpha_{i}}{\left(\rho_{v} \Delta h_{1}\right) R_{1}^{\prime} \theta_{1}}+\frac{\eta_{1} \Delta t}{\left(r h o_{v} \Delta h_{1}\right) \theta_{1}}\right)+\frac{C_{T, 1} C_{p}^{a i r} \rho_{a} \Delta t}{R_{1} \theta_{1}\left(\rho_{v} \Delta h_{1}\right)}+\frac{C_{q, 1} \lambda \rho_{a} \Delta t}{\left(\rho_{v} \Delta h_{1}\right) R_{1}^{\prime} \theta_{1}}\right)
\end{aligned}
$$


and:

$$
\begin{aligned}
& G_{1}=\left(T_{L, 1}^{t}+\frac{\lambda \rho_{a} \Delta t \beta_{i}}{\left(\rho_{v} \Delta h_{1}\right) R_{1}^{\prime} \theta_{1}}+\frac{\eta_{3} R_{S W}^{\text {down }} \Delta t}{\left(\rho_{v} \Delta h_{1}\right) \theta_{1}}\right. \\
& \left.\quad+\frac{\eta_{2} \Delta t}{\left(\rho_{v} \Delta h_{1}\right) \theta_{1}}-\frac{B_{T, 1} C_{p}^{\text {air }} \rho_{a} \Delta t}{R_{1} \theta_{1}\left(\rho_{v} \Delta h_{1}\right)}-\frac{B_{q, 1} \lambda \rho_{a} \Delta t}{\left(\rho_{v} \Delta h_{1}\right) R_{1}^{\prime} \theta_{1}}\right) / \\
& \left(1-\left(\frac{C_{p}^{\text {air }} \rho_{a} \Delta t}{\left(\rho_{v} \Delta h_{1}\right) R_{1} \theta_{1}}+\frac{\lambda \rho_{a} \Delta t \alpha_{i}}{\left(\rho_{v} \Delta h_{1}\right) R_{1}^{\prime} \theta_{1}}+\frac{\eta_{2} \Delta t}{\left(\rho_{v} \Delta h_{1}\right) \theta_{1}}\right)+\frac{C_{T, 1} C_{p}^{a i r} \rho_{a} \Delta t}{R_{1} \theta_{1}\left(\rho_{v} \Delta h_{1}\right)}+\frac{C_{q, 1} \lambda \rho_{a} \Delta t}{\left(\rho_{v} \Delta h_{1}\right) R_{1}^{\prime} \theta_{1}}\right)
\end{aligned}
$$

We now seek to rearrange b) and c) into expressions of the form:

i) $T_{a, 1}^{t+1} X_{1}=X_{2}+\phi_{H}^{t+1} X_{3}+q_{a, 1}^{t+1} X_{4}+T_{\text {leaf }, 1}^{t+1} X_{5}$

and:

ii) $\quad q_{a, 1}^{t+1} Y_{1}=Y_{2}+\phi_{\lambda E}^{t+1} Y_{3}+T_{a, 1}^{t+1} Y_{4}+T_{l e a f, 1}^{t+1} Y_{5}$

The same process as in the previous section means that we can assign $A_{T, 1}, B_{T, 1}, C_{T, 1}$, $D_{T, 1}, A_{q, 1}, B_{q, 1}, C_{q, 1}, D_{q, 1}$ exactly as previously (expressions (S3.37) to (S3.44)), and define $X_{1}$ to $Y_{5}$ as follows:

$$
\begin{aligned}
& X_{1}=1-\Delta t\left(\frac{k_{1} A_{T, 2}}{\Delta z_{1} \Delta h_{1}}-\frac{k_{1} C_{T, 2} F_{2}}{\Delta z_{1} \Delta h_{1}}+\frac{k_{1}}{\Delta z_{1} \Delta h_{1}}+\frac{1}{\Delta h_{1} R_{1}}\right) \\
& X_{2}=T_{a, 1}^{t}+\Delta t\left(\frac{k_{1} B_{T, 2}}{\Delta z_{1} \Delta h_{1}}+\frac{k_{1} C_{T, 2} G_{2}}{\Delta z_{1} \Delta h_{1}}\right) \\
& X_{3}=-\Delta t\left(\frac{1}{\Delta h}\right)\left(\frac{1}{\rho_{a} C_{p}^{a i r}}\right) \\
& X_{4}=\Delta t\left(\frac{k_{1} C_{T, 2} E_{2}+k_{1} D_{T, 2}}{\Delta z_{1} \Delta h_{1}}\right) \\
& X_{5}=\Delta t\left(\frac{1}{\Delta h_{1} R_{1}}\right) \\
& Y_{1}=1-\Delta t\left(\frac{k_{1} A_{q, 2}}{\Delta z_{1} \Delta h_{1}}-\frac{k_{1} C_{q, 2} E_{2}}{\Delta z_{1} \Delta h_{1}}+\frac{1}{\Delta h_{1} R^{\prime}}+\frac{k_{1}}{\Delta z_{1} \Delta h_{1}}\right) \\
& Y_{2}=q_{a, 1}^{t}+\Delta t\left(\frac{k_{1} B_{q, 2}}{\Delta z_{1} \Delta h_{1}}+\frac{k_{1} C_{q, 2} G_{2}}{\Delta z_{1} \Delta h_{1}}+\frac{\beta_{1}}{\Delta h_{1} R_{1}^{\prime}}\right)
\end{aligned}
$$




$$
\begin{aligned}
& Y_{3}=-\Delta t\left(\frac{1}{\Delta h_{1}}\right)\left(\frac{1}{\rho_{a} \lambda}\right) \\
& Y_{4}=\Delta t\left(\frac{k_{1} C_{q, 2} F_{2}}{\Delta z_{1} \Delta h_{1}}+\frac{k_{1} D_{q, 2}}{\Delta z_{1} \Delta h_{1}}\right) \\
& Y_{5}=\Delta t\left(\frac{\alpha_{1}}{\Delta h_{1} R_{1}^{\prime}}\right)
\end{aligned}
$$

Now, for the lower boundary condition we consider the interaction between the lowest atmospheric level (level 1) and the infinitesimal surface layer (level S). Fluxes of the sensible and latent heat from this layer are given, respectively, by:

i) $\phi_{H}^{t+1}=-\left(\rho_{a} C_{p}^{a i r}\right) k_{\text {surf }} \frac{T_{a, 1}^{t+1}-T_{\text {surf }}^{t+1}}{\Delta z_{\text {surf }}}$
ii) $\phi_{\lambda E}^{t+1}=-\left(\rho_{a} \lambda\right) k_{\text {surf }} \frac{q_{a, 1}^{t+1}-q_{\text {surf }}^{t+1}}{\Delta z_{\text {surf }}}$

i) $\quad \phi_{H}^{t+1}=\frac{\rho_{a} C_{p}^{a i r} k_{\text {surf }}}{\Delta z_{\text {surf }}}\left(A_{T, 1} \phi_{H}^{t+1}+B_{T, 1}+C_{T, 1} T_{L, 1}^{t+1}+D_{T, 1} \phi_{\lambda E}^{t+1}-T_{\text {surf }}^{t+1}\right)$

ii) $\quad \phi_{\lambda E}^{t+1}=\frac{\left(\rho_{a} \lambda\right) k_{\text {surf }}}{\Delta z_{\text {surf }}}\left(A_{q, 1} \phi_{\lambda E}^{t+1}+B_{q, 1}+C_{q, 1} T_{L, 1}^{t+1}+D_{q, 1} \phi_{H}^{t+1}-q_{\text {surf }}^{t+1}\right)$

We use a substitution for the leaf temperature:

$T_{L, 1}^{t+1}=E_{1} \phi_{\lambda E}^{t+1}+F_{1} \phi_{H}^{t+1}+G_{1}$

i) $\quad \phi_{H}^{t+1}=-\frac{\left(\rho_{a} C_{p}^{a i r}\right) k_{\text {surf }}}{\Delta z_{\text {surf }}}\left(A_{T, 1} \phi_{H}^{t+1}+B_{T, 1}+C_{T, 1}\left(E_{1} \phi_{\lambda E}^{t+1}+F_{1} \phi_{H}^{t+1}+G_{1}\right)\right.$

$$
\left.+D_{T, 1} \phi_{\lambda E}^{t+1}-T_{s u r f}^{t+1}\right)
$$

ii) $\quad \phi_{\lambda E}^{t+1}=-\frac{\left(\rho_{a} \lambda\right) k_{\text {surf }}}{\Delta z_{\text {surf }}}\left(A_{q, 1} \phi_{\lambda E}^{t+1}+B_{q, 1}+C_{q, 1}\left(E_{1} \phi_{\lambda E}^{t+1}+F_{1} \phi_{H}^{t+1}+G_{1}\right)\right.$

$$
\left.+D_{q, 1} \phi_{H}^{t+1}-q_{\text {surf }}^{t+1}\right)
$$


i) $\quad \phi_{H}^{t+1}\left(1+\frac{\left(\rho_{a} C_{p}^{\text {air }}\right) k_{\text {surf }}}{\Delta z_{\text {surf }}}\left(A_{T, 1}+C_{T, 1} F_{1}\right)=-\frac{\left(\rho_{a} C_{p}^{\text {air }}\right) k_{\text {surf }}}{\Delta z_{\text {surf }}}\left(B_{T, 1}+C_{T, 1} G_{1}-T_{\text {surf }}^{t+1}\right)\right.$

$$
-\frac{\left(\rho_{a} C_{p}^{\text {air }}\right) k_{\text {surf }}}{\Delta z_{\text {surf }}}\left(\phi_{\lambda E}^{t+1}\left(C_{T, 1} E_{1}+D_{T, 1}\right)\right)
$$

ii) $\quad \phi_{\lambda E}^{t+1}\left(1+\frac{\left(\rho_{a} \lambda\right) k_{\text {surf }}}{\Delta z_{\text {surf }}}\left(A_{q, 1}+C_{q, 1} E_{1}\right)\right)=$

$$
\begin{gathered}
-\frac{\left(\rho_{a} \lambda\right) k_{\text {surf }}}{\Delta z_{\text {surf }}}\left(B_{q, 1}+C_{q, 1} G_{1}-q_{\text {surf }}^{t+1}\right) \\
-\frac{\left(\rho_{a} \lambda\right) k_{\text {surf }}}{\Delta z_{\text {surf }}}\left(\phi_{H}^{t+1}\left(C_{q, 1} F_{1}+D_{q, 1}\right)\right)
\end{gathered}
$$

ii) $\phi_{\lambda E}^{t+1}\left(1+\frac{\left(\rho_{a} \lambda\right) k_{\text {surf }}}{\Delta z_{\text {surf }}}\left(A_{q, 1}+C_{q, 1} E_{1}\right)\right)=$

$$
\begin{array}{r}
-\frac{\left(\rho_{a} \lambda\right) k_{\text {surf }}}{\Delta z_{\text {surf }}}\left(B_{q, 1}+C_{q, 1} G_{1}-\left(\alpha_{\text {surf }} T_{\text {surf }}^{t+1}+\beta_{\text {surf }}\right)\right) \\
-\frac{\left(\rho_{a} \lambda\right) k_{\text {surf }}}{\Delta z_{\text {surf }}}\left(\phi_{H}^{t+1}\left(C_{q, 1} F_{1}+D_{q, 1}\right)\right)
\end{array}
$$

and abbreviate to:

$$
\begin{aligned}
& \text { i) } \quad \Omega_{1} \phi_{H}^{t+1}=\Omega_{2}+\Omega_{3} T_{\text {surf }}^{t+1}+\Omega_{4} \phi_{\lambda E}^{t+1} \\
& \text { ii) } \quad \Omega_{5} \phi_{\lambda E}^{t+1}=\Omega_{6}+\Omega_{7} T_{\text {surf }}^{t+1}+\Omega_{8} \phi_{H}^{t+1}
\end{aligned}
$$

where:

$$
\begin{aligned}
& \Omega_{1}=1+\frac{\left(\rho_{a} C_{p}^{\text {air }}\right) k_{\text {surf }}}{\Delta z_{\text {surf }}}\left(A_{T, 1}+C_{T, 1} F_{1}\right) \\
& \Omega_{2}=-\frac{\left(\rho_{a} C_{p}^{\text {air }}\right) k_{\text {surf }}}{\Delta z_{\text {surf }}}\left(B_{T, 1}+C_{T, 1} G_{1}\right) \\
& \Omega_{3}=\frac{\rho_{a} C_{p}^{\text {air }} k_{\text {surf }}}{\Delta z_{\text {surf }}} \\
& \Omega_{4}=-\frac{\left(\rho_{a} C_{p}^{\text {air }} k_{\text {surf }}\right)}{\Delta z_{\text {surf }}}\left(C_{T, 1} E_{1}+D_{T, 1}\right) \\
& \Omega_{5}=1+\frac{\left(\rho_{a} \lambda\right) k_{\text {surf }}}{\Delta z_{\text {surf }}}\left(A_{q, 1}+C_{q, 1} E_{1}\right) \\
& \Omega_{6}=-\frac{\left(\rho_{a} \lambda\right) k_{\text {surf }}}{\Delta z_{\text {surf }}}\left(B_{q, 1}+C_{q, 1} G_{1}-\beta_{\text {surf }}\right)
\end{aligned}
$$




$$
\begin{aligned}
& \Omega_{7}=\frac{\left(\rho_{a} \lambda\right) k_{\text {surf }}}{\Delta z_{\text {surf }}} \alpha_{\text {surf }} \\
& \Omega_{8}=-\frac{\left(\rho_{a} \lambda\right) k_{\text {surf }}}{\Delta z_{\text {surf }}}\left(C_{q, 1} F_{1}+D_{q, 1}\right)
\end{aligned}
$$

cross substitute:

$$
\begin{array}{ll}
\text { i) } & \Omega_{1} \phi_{H}^{t+1}=\Omega_{2}+\Omega_{3} T_{\text {surf }}^{t+1}+\frac{\Omega_{4}}{\Omega_{5}}\left(\Omega_{6}+\Omega_{7} T_{\text {surf }}^{t+1}+\Omega_{8} \phi_{H}^{t+1}\right) \\
\text { i) } & \phi_{H}^{t+1}\left(\Omega_{1}-\frac{\Omega_{4}}{\Omega_{5}} \Omega_{8}\right)=\left(\Omega_{2}+\frac{\Omega_{4}}{\Omega_{5}} \Omega_{6}\right)+T_{\text {surf }}^{t+1}\left(\Omega_{3}+\frac{\Omega_{4} \Omega_{7}}{\Omega_{5}}\right)
\end{array}
$$

and:
ii) $\quad \Omega_{5} \phi_{\lambda E}^{t+1}=\Omega_{6}+\Omega_{7} T_{\text {surf }}^{t+1}+\frac{\Omega_{8}}{\Omega_{1}}\left(\Omega_{2}+\Omega_{3} T_{\text {surf }}^{t+1}+\Omega_{4} \phi_{\lambda E}^{t+1}\right)$
ii) $\quad \phi_{\lambda E}^{t+1}\left(\Omega_{5}-\frac{\Omega_{8}}{\Omega_{1}} \Omega_{4}\right)=\left(\Omega_{6}+\frac{\Omega_{8}}{\Omega_{1}} \Omega_{2}\right)+T_{\text {surf }}^{t+1}\left(\Omega_{7}+\frac{\Omega_{8} \Omega_{3}}{\Omega_{1}}\right)$

Now we abbreviate $\Omega$ by substituting for $\xi$ :

$$
\begin{aligned}
& \xi_{1}=\frac{\Omega_{2}+\frac{\Omega_{4}}{\Omega_{5}} \Omega_{6}}{\Omega_{1}-\frac{\Omega_{4}}{\Omega_{5}} \Omega_{8}} \\
& \xi_{2}=\frac{\Omega_{3}+\frac{\Omega_{4} \Omega_{7}}{\Omega_{5}}}{\Omega_{1}-\frac{\Omega_{4}}{\Omega_{5}} \Omega_{8}} \\
& \xi_{3}=\frac{\Omega_{6}+\frac{\Omega_{8} \Omega_{2}}{\Omega_{1}}}{\Omega_{5}-\frac{\Omega 8 \Omega_{4}}{\Omega_{1}}} \\
& \xi_{4}=\frac{\Omega_{7}+\frac{\Omega_{8} \Omega_{3}}{\Omega_{1}}}{\Omega_{5}-\frac{\Omega_{8} \Omega_{4}}{\Omega_{1}}}
\end{aligned}
$$

And we can equations $i$ ) and $i i$ ) in terms of $\xi$ :

$$
\begin{aligned}
\text { i) } \quad \phi_{H}^{t+1}=\xi_{1}+\xi_{2} T_{\text {surf }}^{t+1} \\
\text { ii) } \phi_{\lambda E}^{t+1}=\xi_{3}+\xi_{4} T_{\text {surf }}^{t+1} \\
T_{\text {surf }}^{t+1}=T_{\text {surf }}^{t}+\frac{\Delta t}{\theta_{0}}\left(\left(R_{L W, \text { surf }}+R_{S W, \text { surf }}+\xi_{1}+\xi_{2} T_{\text {surf }}^{t+1}+\xi_{3}+\xi_{4} T_{\text {surf }}^{t+1}\right)-J_{\text {soil }}\right)
\end{aligned}
$$




$$
T_{\text {surf }}^{t+1}\left(1-\xi_{2} \frac{\Delta t}{\theta_{0}}-\xi_{4} \frac{\Delta t}{\theta_{0}}\right)=T_{\text {surf }}^{t}+\frac{\Delta t}{\theta_{0}}\left(R_{L W, \text { surf }}+R_{S W, \text { surf }}+\xi_{1}+\xi_{3}-J_{\text {soil }}\right)
$$

and so:

$$
T_{\text {surf }}^{t+1}=\frac{T_{\text {surf }}^{t}+\frac{\Delta t}{\theta_{0}}\left(R_{L W, \text { surf }}+R_{S W, \text { surf }}+\xi_{1}+\xi_{3}-J_{\text {soil }}\right)}{\left(1-\xi_{2} \frac{\Delta t}{\theta_{0}}-\xi_{4} \frac{\Delta t}{\theta_{0}}\right)}
$$

We therefore have an expression for the surface temperature $T_{\text {surf }}^{t+1}$, in terms of the downwelling radiation that is incident on the surface $\left(R_{L W}\right.$ and $\left.R_{S W}\right)$, the heat capacity of the infinitesimal surface layer $\left(\theta_{0}\right)$, the vegetation layer directly above the surface $\left(\xi_{1}\right.$, $\xi_{2}, \xi_{3}$ and $\left.\xi_{4}\right)$ and the heat from the soil system $\left(J_{\text {soil }}\right)$.

The radiation that is received by the lowermost level is provided by the radiation scheme.

So to re-write the above equation including the factors $\eta_{1, S}, \eta_{2, S}$ and $\eta_{3, S}$ :

$$
T_{\text {surf }}^{t+1}=\frac{T_{\text {surf }}^{t}+\frac{\Delta t}{\theta_{0}}\left(\eta_{2, S}+\eta_{3, S} R_{S W}^{\text {down }}+\xi_{1}+\xi_{3}\right)-J_{\text {soil }}}{\left(1-\frac{\Delta t}{\theta_{0}}\left(\xi_{2}+\xi_{4}+\eta_{1, S}\right)\right)}
$$




\section{References}

Best, M. J., Beljaars, A. C. M., Polcher, J. and Viterbo, P. (2004), 'A proposed structure for coupling tiled surfaces with the planetary boundary layer', Journal of Hydrometeorology 5, 1271-1278.

Dufresne, J.-L. and Ghattas, J. (2009), Description du schema de la couche limite turbulente et linterface avec la surface planetaire dans LMDZ, Technical report.

$\mathrm{Gu}$, L. (1988), Longwave radiative transfer in plant canopies, $\mathrm{PhD}$ thesis, University of Virginia.

Gu, L., Shugart, H. H., Fuentes, J. D., Black, T. A. and Shewchuk, S. R. (1999), 'Micrometeorology, biophysical exchanges and NEE decomposition in a two-storey boreal forest - development and test of an integrated model', Agricultural and Forest Meteorology 94, 123-148.

Makar, P. A., Fuentes, J. D., Wang, D., Staebler, R. M. and Wiebe, H. A. (1999), 'Chemical processing of biogenic hydrocarbons within and above a temperate deciduous forest', Journal of Geophysical Research 104(D3), 3581-3603.

URL: http://www.agu.org/pubs/crossref/1999/1998JD100065.shtml

McGrath, M. J., Pinty, B., Ryder, J., Otto, J. and Luyssaert, S. (2015), 'A multilevel canopy radiative transfer scheme based on a domain-averaged structure factor'.

Monteith, J. and Unsworth, M. H. (2008), Principles of Environmental Physics, Elsevier.

Ogée, J., Brunet, Y., Loustau, D., Berbigier, P. and Delzon, S. (2003), 'MuSICA, a CO2, water and energy multilayer, multileaf pine forest model: evaluation from hourly to yearly time scales and sensitivity analysis', Global Change Biology 9(5), 697-717.

URL: http://doi.wiley.com/10.1046/j.1365-2486.2003.00628.x

Pinty, B., Lavergne, T., Dickinson, R. E., Widlowski, J.-L., Gobron, N. and Verstraete, M. M. (2006), 'Simplifying the interaction of land surfaces with radiation for relating remote sensing products to climate models', Journal of Geophysical Research 111(D2), 1-20.

URL: http://www.agu.org/pubs/crossref/2006/2005JD005952.shtml

Raupach, M. R. (1989), 'Applying Lagrangian fluid mechanics to infer scalar source distributions from concentration profiles in plant canopies', Agricultural and Forest Meteorology 47, 85-108.

Richtmyer, R. D. and Morton, K. W. (1967), Difference Methods for Initial-Value Problems (second edition), Wiley-Interscience.

Vieno, M. (2006), The use of an atmospheric chemistry-transport model (FRAME) over the UK and the development of its numerical and physical schemes, PhD thesis, University of Edinburgh. 


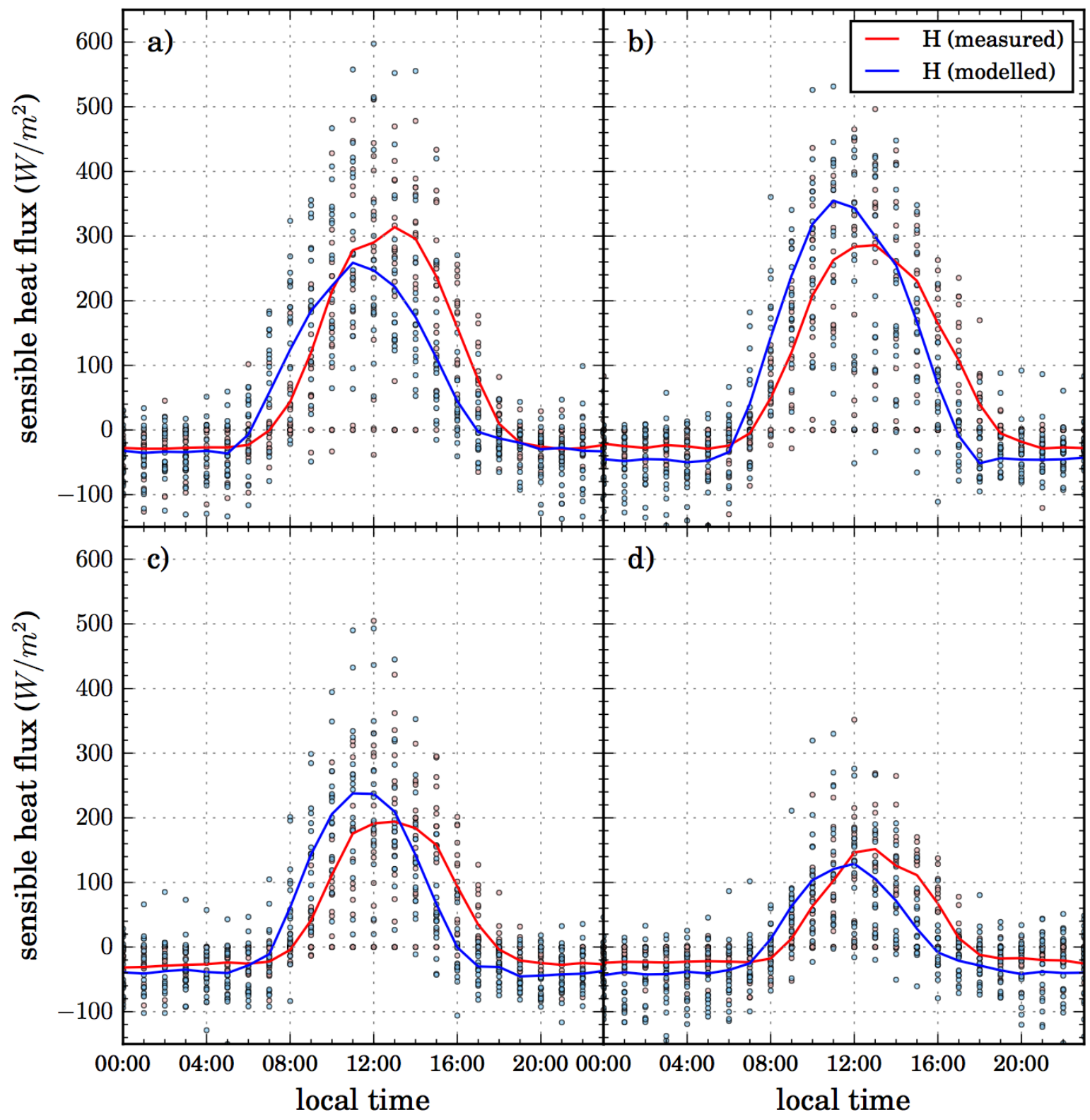

Figure S1: The analysis of Figure 3 is repeated for the four seasons of the year (every 5th measurement is shown in the background in a lighter colour). Hourly average sensible heat flux (annual average): a) spring; b) summer; c) autumn; d) winter 


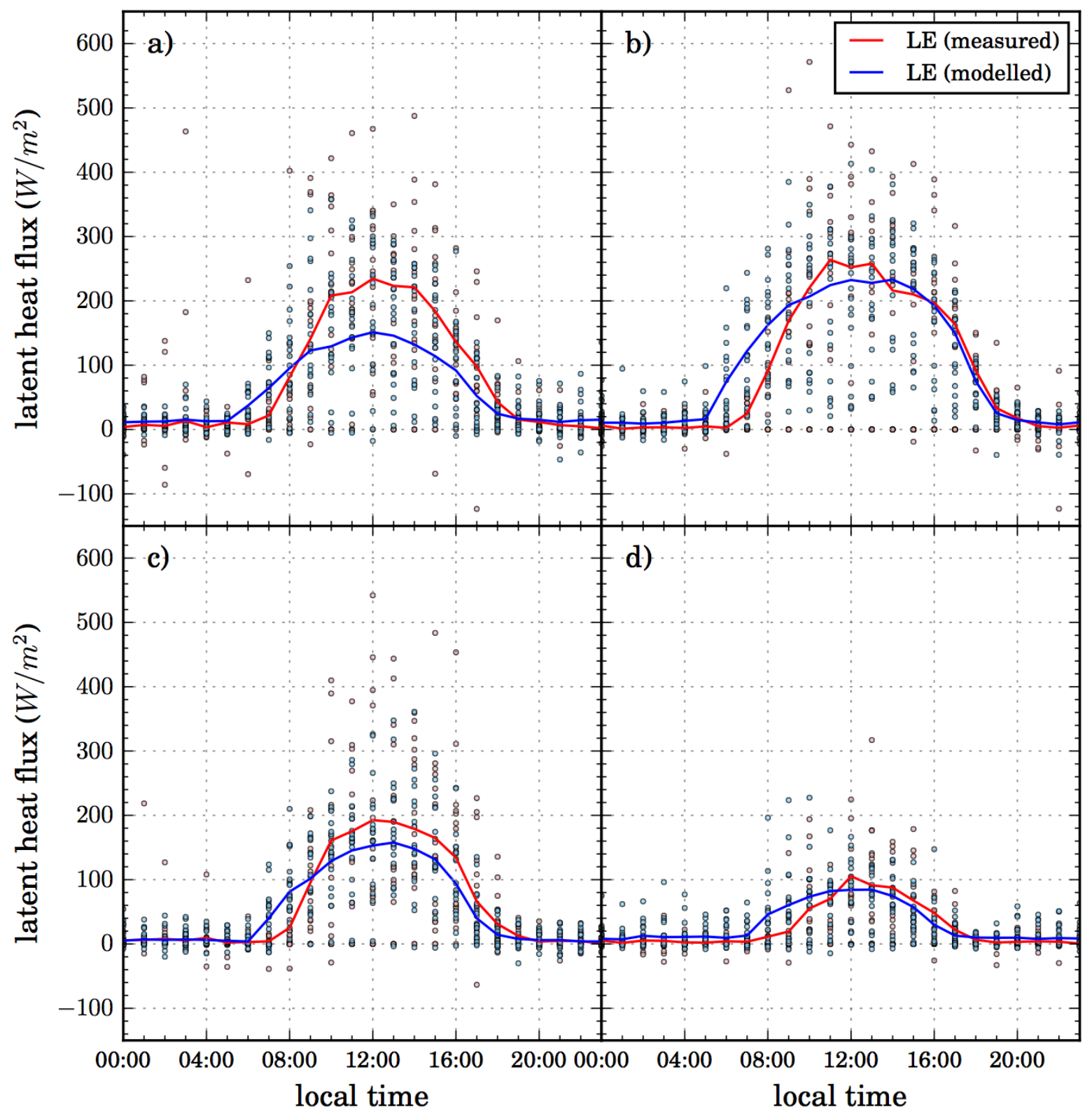

Figure S2: The analysis of Figure 3 repeated for the four seasons of the year (every 5th measurement is shown in the background in a lighter colour). hourly latent sensible heat flux (annual average): a) spring; b) summer; c) autumn; d) winter 